\title{
SAI: Safety Application Identifier Algorithm at MAC Layer for Vehicular Safety Message Dissemination Over LTE VANET Networks
}

\author{
Shuja Ansari $\left(\mathbb{D},{ }^{1}\right.$ Marvin Sánchez, ${ }^{1}$ Tuleen Boutaleb $\mathbb{D},{ }^{1}$ Sinan Sinanovic $\mathbb{D},{ }^{1}$ \\ Carlos Gamio, ${ }^{1}$ and Ioannis Krikidis ${ }^{2}$ \\ ${ }^{1}$ School of Engineering and Built Environment, Glasgow Caledonian University, Glasgow, UK \\ ${ }^{2}$ Department of Electrical and Computer Engineering, Faculty of Engineering, University of Cyprus, Nicosia, Cyprus
}

Correspondence should be addressed to Shuja Ansari; shuja.ansari@gcu.ac.uk

Received 25 August 2017; Revised 4 January 2018; Accepted 30 January 2018; Published 27 February 2018

Academic Editor: Orhan Gazi

Copyright (C) 2018 Shuja Ansari et al. This is an open access article distributed under the Creative Commons Attribution License, which permits unrestricted use, distribution, and reproduction in any medium, provided the original work is properly cited.

\begin{abstract}
Vehicular safety applications have much significance in preventing road accidents and fatalities. Among others, cellular networks have been under investigation for the procurement of these applications subject to stringent requirements for latency, transmission parameters, and successful delivery of messages. Earlier contributions have studied utilization of Long-Term Evolution (LTE) under single cell, Friis radio, or simplified higher layer. In this paper, we study the utilization of LTE under multicell and multipath fading environment and introduce the use of adaptive awareness range. Then, we propose an algorithm that uses the concept of quality of service (QoS) class identifiers (QCIs) along with dynamic adaptive awareness range. Furthermore, we investigate the impact of background traffic on the proposed algorithm. Finally, we utilize medium access control (MAC) layer elements in order to fulfill vehicular application requirements through extensive system-level simulations. The results show that, by using an awareness range of up to $250 \mathrm{~m}$, the LTE system is capable of fulfilling the safety application requirements for up to 10 beacons/s with 150 vehicles in an area of $2 \times 2 \mathrm{~km}^{2}$. The urban vehicular radio environment has a significant impact and decreases the probability for end-toend delay to be $\leq 100 \mathrm{~ms}$ from $93 \%-97 \%$ to $76 \%-78 \%$ compared to the Friis radio environment. The proposed algorithm reduces the amount of vehicular application traffic from $21 \mathrm{Mbps}$ to $13 \mathrm{Mbps}$, while improving the probability of end-to-end delay being $\leq 100 \mathrm{~ms}$ by $20 \%$. Lastly, use of MAC layer control elements brings the processing of messages towards the edge of network increasing capacity of the system by about $50 \%$.
\end{abstract}

\section{Introduction}

Cellular Networks are ubiquitous technologies that have evolved in order to satisfy the continuous increasing traffic and quality of service (QoS) demands required by existing and future user applications. Operators currently utilize one or several standardized technologies as the application market's demand increases. For instance, voice and data applications requiring low latency can be served by wideband code division multiple access (WCDMA) while higher data rates with lower latency can be provided by introducing evolved universal mobile telecommunications service terrestrial radio access networks (E-UTRAN, commercially referred to as Long-Term Evolution (LTE)) [1].
Vehicular communications presiding under the umbrella of cooperative intelligent transportation systems (C-ITS) sets forth stringent requirements in terms of latency and successful packet delivery. There are mainly three applications in vehicular networks as categorized by [2]: safety (e.g., cooperative forward collision warning, to avoid rearend collisions), transport efficiency (e.g., traffic light optimal speed advisory, to assist the driver to arrive during a green phase), and information/entertainment (e.g., including remote wireless diagnosis, to make the state of the vehicle accessible for remote diagnosis). Safety applications, also referred to as driver safety applications, are envisioned to help prevent fatalities on the road by sending periodic messages (beacons) in the vehicles neighborhood. These applications 
TABLE 1: Safety application requirements.

\begin{tabular}{|c|c|c|c|c|c|}
\hline Safety application & Message type & Communication mode & Minimum frequency & $\begin{array}{l}\text { Critical } \\
\text { latency }\end{array}$ & $\begin{array}{c}\text { Transmission } \\
\text { range }\end{array}$ \\
\hline $\begin{array}{l}\text { Intersection collision } \\
\text { warning }\end{array}$ & CAM & $\begin{array}{c}\text { Broadcasting periodic } \\
\text { messages }\end{array}$ & $10 \mathrm{~Hz}$ & $\sim 100 \mathrm{~ms}$ & $150 \mathrm{~m}$ \\
\hline Lane change assistance & CAM & $\begin{array}{l}\text { Cooperation awareness } \\
\text { between vehicles }\end{array}$ & $10 \mathrm{~Hz}$ & $\sim 100 \mathrm{~ms}$ & $150 \mathrm{~m}$ \\
\hline Slow vehicle indication & CAM/DENM & Broadcasting state periodically & $2 \mathrm{~Hz}$ & $\sim 100 \mathrm{~ms}$ & $200 \mathrm{~m}$ \\
\hline $\begin{array}{l}\text { Traffic light speed } \\
\text { advisory/violation }\end{array}$ & CAM/DENM & $\begin{array}{c}\text { Broadcasting periodic } \\
\text { messages }\end{array}$ & $2 \mathrm{~Hz}$ & $\sim 100 \mathrm{~ms}$ & $150 \mathrm{~m}$ \\
\hline Overtaking vehicle warning & CAM & Broadcasting overtaking state & $10 \mathrm{~Hz}$ & $\sim 100 \mathrm{~ms}$ & $300 \mathrm{~m}$ \\
\hline Head on collision warning & CAM & $\begin{array}{c}\text { Broadcasting periodic } \\
\text { messages }\end{array}$ & $10 \mathrm{~Hz}$ & $\sim 100 \mathrm{~ms}$ & $200 \mathrm{~m}$ \\
\hline Collision risk warning & CAM/DENM & $\begin{array}{l}\text { Time limited periodic } \\
\text { messages on event }\end{array}$ & $10 \mathrm{~Hz}$ & $\sim 100 \mathrm{~ms}$ & $300-500 \mathrm{~m}$ \\
\hline $\begin{array}{l}\text { Cooperative forward } \\
\text { collision warning }\end{array}$ & CAM & $\begin{array}{l}\text { Cooperation awareness } \\
\text { between vehicles }\end{array}$ & $10 \mathrm{~Hz}$ & $\sim 100 \mathrm{~ms}$ & $150 \mathrm{~m}$ \\
\hline Emergency vehicle warning & CAM/DENM & $\begin{array}{c}\text { Broadcasting periodic } \\
\text { messages }\end{array}$ & $10 \mathrm{~Hz}$ & $\sim 100 \mathrm{~ms}$ & $300 \mathrm{~m}$ \\
\hline $\begin{array}{l}\text { Cooperative merging } \\
\text { assistance }\end{array}$ & CAM & $\begin{array}{l}\text { Cooperation awareness } \\
\text { between vehicles }\end{array}$ & $1 \mathrm{~Hz}$ & $\sim 1000 \mathrm{~ms}$ & $250 \mathrm{~m}$ \\
\hline Speed limits notification & CAM & $\begin{array}{l}\text { Broadcasting periodic } \\
\text { messages }\end{array}$ & $1-10 \mathrm{~Hz}$ & $\sim 100 \mathrm{~ms}$ & $300 \mathrm{~m}$ \\
\hline $\begin{array}{l}\text { Motorcycle approaching } \\
\text { indication }\end{array}$ & CAM & $\begin{array}{l}\text { Cooperation awareness } \\
\text { between vehicles }\end{array}$ & $2 \mathrm{~Hz}$ & $\sim 100 \mathrm{~ms}$ & $150 \mathrm{~m}$ \\
\hline
\end{tabular}

and technologies have been subject of much research since the allocation of the dedicated short-range communication (DSRC) licensed spectrum on $5.9 \mathrm{GHz}$ frequency band [3]. In order to utilize the DSRC spectrum, equipment must be compliant with the IEEE wireless access in vehicular environment (WAVE) standards suite that includes IEEE 802.11p designed for vehicular environment. Utilizing this standard, vehicular ad hoc networks (VANET) can be created on roads having the advantage of working without the need of any fixed infrastructure, known as vehicle-to-vehicle (V2V) communications, or with the installation of road side units (RSUs); the latter can be used for vehicle-to-infrastructure (V2I) communications, to extend the type of services and applications.

Provisioning of V2I using IEEE 802.11p requires installation of RSUs, which means deployment and maintenance of a dedicated network is required $[4,5]$. This has motivated several authors to propose the utilization of LTE as an alternative to provide V2I applications and furthermore investigate if LTE can satisfy the stringent requirements with V2V applications $[6,7]$.

In continuation of the previously contributed work from various authors described in Section 1.1, contributions of this paper include the following:

(i) Analysis of intercell interference and handover impact on latency of the end-to-end safety systems and studying the impact of vehicular urban radio environment employing multipath fading channels under European (Glasgow) and American (Manhattan) mobility traces (ii) Investigation of variable awareness range that can be used over LTE to fulfill various vehicular application requirements (see Table 1 )

(iii) Investigation of regular cellular traffic's impact on vehicular applications running on LTE networks

(iv) Proposal of safety application identifier (SAI) algorithm utilizing dynamic selection of transmission parameters within the impact of the above-mentioned contributions

(v) Utilization of medium access control (MAC) layer control elements with the proposed SAI in order to reduce latency of the network, while increasing capacity for vehicular application use.

In light of these contributions, related literature is presented in the following section.

1.1. Related Works. When utilizing LTE, uplink transmissions from vehicles to a centralized server are commonly done using unicast [9]. For the downlink, forwarding of the received information can be delivered to multiple vehicles using either unicast, broadcast, or multicast transmissions. Broadcast/multicast requires transmissions to be received by users in the worst cell quality conditions (e.g., at the cell edge) and results in lower average spectral efficiency (bps/Hz) compared to unicast transmissions [1, Chapter 1]. In spite of this, if the number of receiving users of the message is larger than the spectral efficiency reduction relative to unicast, it is more efficient to use broadcast/multicast transmissions in order to cater capacity blocks. 
Broadcast and unicast transmissions with LTE have been investigated in [10-12]. Vinel [10] used a simplified model in order to compute analytically the packet delivery ratio (PDR), assuming fixed number of neighbors (50 vehicles) in a time division duplex (TDD) LTE single cell system. Due to the overall system complexity of LTE, system-level simulations have become an indispensable tool for predicting the performance over more realistic scenarios. In [11], simulations were used to compare IEEE 802.11p with frequency division duplex (FDD) LTE single cell system performance using Friis radio propagation environment on a Manhattan gird mobility model. Their performance evaluation showed that LTE is suitable for VANETs; however, the use of single cell, simplified higher layers, and Friis radio environment does not test the system under extreme propagation scenarios. When it comes to system-level simulations, choice of mobility models carries much importance. Grzybek et al. in [13] studied and compared various mobility generators for VANETs, proposing a mobility generator for realistic traces called vehilux. This generates traces considering the spatial, temporal, and behavioral aspects of traffic distribution. Since the scope of this paper is within the transmission requirements of safety applications, the trace generator adopted is routes mobility model [14]. This generates traces with geographic restrictions on top of spatial, temporal, and behavioral aspects, allowing a fixed number of vehicles in the area throughout the simulation time.

Kato et al. [15] defined the concept of data freshness and used this parameter to evaluate the performance of LTE networks with VANETs. If a vehicle application transmits a packet at a frequency of $10 \mathrm{~Hz}$, that is a message is transmitted every $100 \mathrm{~ms}$, the freshness requirement for that specific application would be $100 \mathrm{~ms}$. They observed that it is possible to achieve a freshness of $100 \mathrm{~ms}$ by having vehicular application server at the edge of LTE network, hence discarding the message if it arrives later than the beacon interval time. Authors evaluated the impact of server location by calculating the amount of network usage. They concluded that, by reducing the round trip time (RTT) of the system, network usage can be reduced to half, hence by placing the server close to the eNodeB or even the LTE core, it can have a significant impact on the scalability of the system.

In order to utilize broadcast functionality on LTE, operators must implement multimedia broadcast multicast services (MBMS) [1, Chapter 14]. Without MBMS, currently only low rate public warning system (PWS) messages can be sent through the broadcast capability of LTE $[16,17]$. Hence, in some situations, unicast is the only available scheme. For instance, the experimental study in [6] used TCP/IP ping application to measure the end-to-end delay in order to evaluate the suitability of LTE for VANET. Also, unicast for cooperative awareness message (CAM) transmissions in multicell environment with LTE has been studied in [12]. In that study, it was found that the downlink was the bottleneck due to high traffic load. However, the study was oriented to determine the capacity limit; therefore, the downlink traffic was modeled by fixed size periodic packet transmission; the handover effect was neglected and did not include the endto-end system. Previous performance evaluations have also suggested that the use of LTE for vehicular communications is suitable, but, without any centralization, it can put enormous load on the network [18]. In the pursuit of centralizing vehicular communications in LTE, group formation and MBMS implementation have been proposed in [18, 19]. Group formation or clustering has shown promising performance. However, clustering relies on relaying transmissions which can pose a privacy and security issue [20].

3GPP release-12 [21] includes device-to-device (D2D) communication under LTE-Advanced which can be a potential candidate technology for V2V communications. Bazzi [22] investigated the use of LTE direct communications (D2D) using full duplex radios in order to enable vehicles to receive and transmit at the same time. Results from their developed analytical framework showed promising reduction of LTE uplink resources as compared with using unicast and broadcast. However, the proposed framework exhibits minimum interference only when the awareness range is set to $100 \mathrm{~m}$. In [23], authors have proposed a scheme that utilizes an unused 16-bit long MAC Control Element inside the buffer status report (BSR) request for the network to identify the receiver and transmitter. This element uses the space reserved for future use and is indexed in the MAC Protocol Data Unit (PDU) subheader by the logical channel identifier (LCID). By doing so, packets sent from the transmitter get to the receiver via $\mathrm{eNodeB}$ instead of traversing through the evolved packet core (EPC), saving the round trip time from the LTE core network $(\mathrm{CN})$ to the access network. A similar concept has been adopted in our work, exploring the possibility of using MAC control elements for message dissemination within vehicular networks in order to reduce latency.

The remainder of this paper is organized as follows: in Section 1.2, the vehicular safety requirements and awareness range are introduced, followed by the proposed algorithm in Section 2; the system model used for LTE along with the performance measures is then included in Section 3; finally, the simulation results and conclusions are discussed in Sections 4 and 5, respectively.

1.2. Vehicular Safety Applications. In vehicular safety applications, the consequence of failure in message delivery within a minimum delay and awareness range may result in a fatal accident [24]. In Table 1, we show a survey of vital active road safety applications along with their requirements for critical latency, beacon frequency, and transmission range [25-29].

Overall, these applications require a critical latency less than or equal to $100 \mathrm{~ms}$ and they differ in the message type, minimum frequency, and transmission range requirements. Without any broadcast routing algorithms, the packet delivery rate for VANETs is generally low ranging between $60 \%$ and $80 \%$ [30]. However, the standards do not define an acceptable packet delivery rate.

Awareness range, previously elaborated in $[9,22,31]$, is the geographical area around the vehicle, where all the neighbors are to be made cognizant of the vehicle. Depending on the applicability of the message this range can vary. There are two types of messages involved in VANETs, classified as CAMs [26] and Decentralized Environment Notification 
TABLE 2: Safety application requirements and SAIs.

\begin{tabular}{|c|c|c|c|c|c|c|}
\hline Safety application & Message type & Transmitted data & $\begin{array}{l}\text { Critical } \\
\text { latency }\end{array}$ & $\begin{array}{l}\text { Transmission } \\
\text { frequency BF }\end{array}$ & $\begin{array}{l}\text { Transmission } \\
\text { range } R\end{array}$ & SAI \\
\hline $\begin{array}{l}\text { Intersection collision } \\
\text { warning }\end{array}$ & CAM & $\begin{array}{l}\text { Vehicle type, position, heading, velocity, } \\
\text { acceleration, yaw rate }\end{array}$ & $\sim 100 \mathrm{~ms}$ & $10 \mathrm{~Hz}$ & $150 \mathrm{~m}$ & \multirow{3}{*}{1} \\
\hline Lane change assistance & CAM & $\begin{array}{l}\text { Position, heading, velocity, acceleration, } \\
\text { turn signal status }\end{array}$ & $\sim 100 \mathrm{~ms}$ & $10 \mathrm{~Hz}$ & $150 \mathrm{~m}$ & \\
\hline $\begin{array}{l}\text { Cooperative forward } \\
\text { collision warning }\end{array}$ & CAM & $\begin{array}{l}\text { Vehicle type, position, heading, velocity, } \\
\text { acceleration, yaw rate }\end{array}$ & $\sim 100 \mathrm{~ms}$ & $10 \mathrm{~Hz}$ & $150 \mathrm{~m}$ & \\
\hline Slow vehicle indication & CAM/DENM & $\begin{array}{l}\text { Vehicle type, position, heading, } \\
\text { acceleration, velocity }\end{array}$ & $\sim 100 \mathrm{~ms}$ & $2 \mathrm{~Hz}$ & $200 \mathrm{~m}$ & 2 \\
\hline $\begin{array}{l}\text { Traffic light speed } \\
\text { advisory/violation }\end{array}$ & CAM/DENM & $\begin{array}{l}\text { Signal phase, timing, position, direction, } \\
\text { road geometry }\end{array}$ & $\sim 100 \mathrm{~ms}$ & $2 \mathrm{~Hz}$ & $150 \mathrm{~m}$ & \multirow{2}{*}{3} \\
\hline $\begin{array}{l}\text { Motorcycle approaching } \\
\text { indication }\end{array}$ & CAM & Vehicle type, position, heading, velocity & $\sim 100 \mathrm{~ms}$ & $2 \mathrm{~Hz}$ & $150 \mathrm{~m}$ & \\
\hline $\begin{array}{l}\text { Overtaking vehicle } \\
\text { warning }\end{array}$ & CAM & Position, velocity, yaw rate, acceleration & $\sim 100 \mathrm{~ms}$ & $10 \mathrm{~Hz}$ & $300 \mathrm{~m}$ & 4 \\
\hline $\begin{array}{l}\text { Head on collision } \\
\text { warning }\end{array}$ & CAM & $\begin{array}{l}\text { Vehicle type, position, heading, velocity, } \\
\text { acceleration, yaw rate }\end{array}$ & $\sim 100 \mathrm{~ms}$ & $10 \mathrm{~Hz}$ & $200 \mathrm{~m}$ & 5 \\
\hline Collision risk warning & CAM/DENM & $\begin{array}{l}\text { Vehicle type, position, heading, velocity, } \\
\text { acceleration, yaw rate }\end{array}$ & $\sim 100 \mathrm{~ms}$ & $10 \mathrm{~Hz}$ & $300-500 \mathrm{~m}$ & 6 \\
\hline $\begin{array}{l}\text { Emergency vehicle } \\
\text { warning }\end{array}$ & CAM/DENM & Position, heading, velocity, acceleration & $\sim 100 \mathrm{~ms}$ & $2 \mathrm{~Hz}$ & $300 \mathrm{~m}$ & 7 \\
\hline $\begin{array}{l}\text { Cooperative merging } \\
\text { assistance }\end{array}$ & CAM & $\begin{array}{l}\text { Curve location, curvature, slope, speed } \\
\text { limit, surface }\end{array}$ & $\sim 1000 \mathrm{~ms}$ & $1 \mathrm{~Hz}$ & $250 \mathrm{~m}$ & 8 \\
\hline Speed limits notification & CAM & $\begin{array}{l}\text { Velocity, acceleration, position, speed } \\
\text { limit, heading }\end{array}$ & $\sim 100 \mathrm{~ms}$ & $1-10 \mathrm{~Hz}$ & $300 \mathrm{~m}$ & 9 \\
\hline
\end{tabular}

Message (DENMs) [27]. CAMs are periodic messages that provide information like the presence, position, and sensor information and are expected to be received by all the vehicles within the awareness range. DENMs are used for cooperative road hazard warnings that are broadcasted in their relevance area whenever a hazardous event occurs [32]. The work in [33] proposes the use of dynamic adaptation of communication radius in order to maximize sum rate; we adopt the same concept and apply it to dynamically adapting communication range in order to minimize end-to-end delay. Figure 1 illustrates the awareness range, $R$, for vehicle $i$. Notice that vehicle $k$ is within the awareness range of $i$ since the distance $d_{i k} \leq R$.

\section{Safety Application Identifier Algorithm}

Message delivery and reliability are a major concern in vehicular networks, due to which a differentiated QoS mechanism is proposed. This mechanism works on the principle of indexing various applications according to their requirements, motivated from QoS class identifier (QCI) implemented in LTE networks [34]. In Table 2, each application from Table 1 is categorized and assigned an SAI. This SAI, which is number ranging between 1 and 9, is included in the transmitted packet appended before the IP header by the application layer as shown in Figure 2. The categorization and assignment of SAI for our model are further discussed in Section 3. This concept is implemented in the form of an algorithm proposed in the following subsection.

\begin{tabular}{|c|}
\hline $\begin{array}{l}\text { Input: VSMs: Vehicles } \rightarrow \text { Server } \\
\text { Output: VSMs: Server } \rightarrow \text { Vehicles } \\
\text { System Setup: } \\
\text { (1) } \text { while Server } \leftarrow \mathrm{VSM}_{i} \text { do } \\
(2) \quad \text { Server locates all vehicles } \\
(3) \quad \mathrm{VSM}_{i} \rightarrow\left(\mathrm{SAI}_{i}, \text { Position }\left(d_{k}\right)\right) \\
(4) \quad \mathrm{SAI}_{i} \rightarrow\left(R_{i}, \mathrm{BF}_{i}\right) \\
(5) \quad\left(R_{i}, d_{k}\right) \rightarrow \text { Distance }\left(d_{i k}\right) \\
(6) \\
\mathbf{F}_{i}=\left\{\forall k: d_{i k}<R_{i}, i \neq k\right\}, \\
(7) \quad \text { Server } \Rightarrow \text { Vehicles } \in \mathbf{F}_{i} \text { at } \mathrm{BF}_{i} \\
(8) \text { end while } \\
(9) \text { return } \mathbf{F}_{i}\end{array}$ \\
\hline
\end{tabular}

Algorithm 1: SAI Algorithm.

2.1. SAI Algorithm. With the concept of safety application indexing, we propose an algorithm that does not include the use of group formation or IEEE 802.11p. The proposed algorithm is a process that is carried out on the vehicular safety application (VSA) server elaborated in Algorithm 1. When the vehicles start transmissions, the packet is carried from the eNodeB to the VSA server via the CN. This packet contains vehicle location $\left(d_{k}\right)$ and corresponding vehicular safety message (VSM). As soon as the server starts receiving these messages, it locates all the vehicles being served by the network, forming a virtual geographical map of the served area. The VSA server, while receiving the packets, 


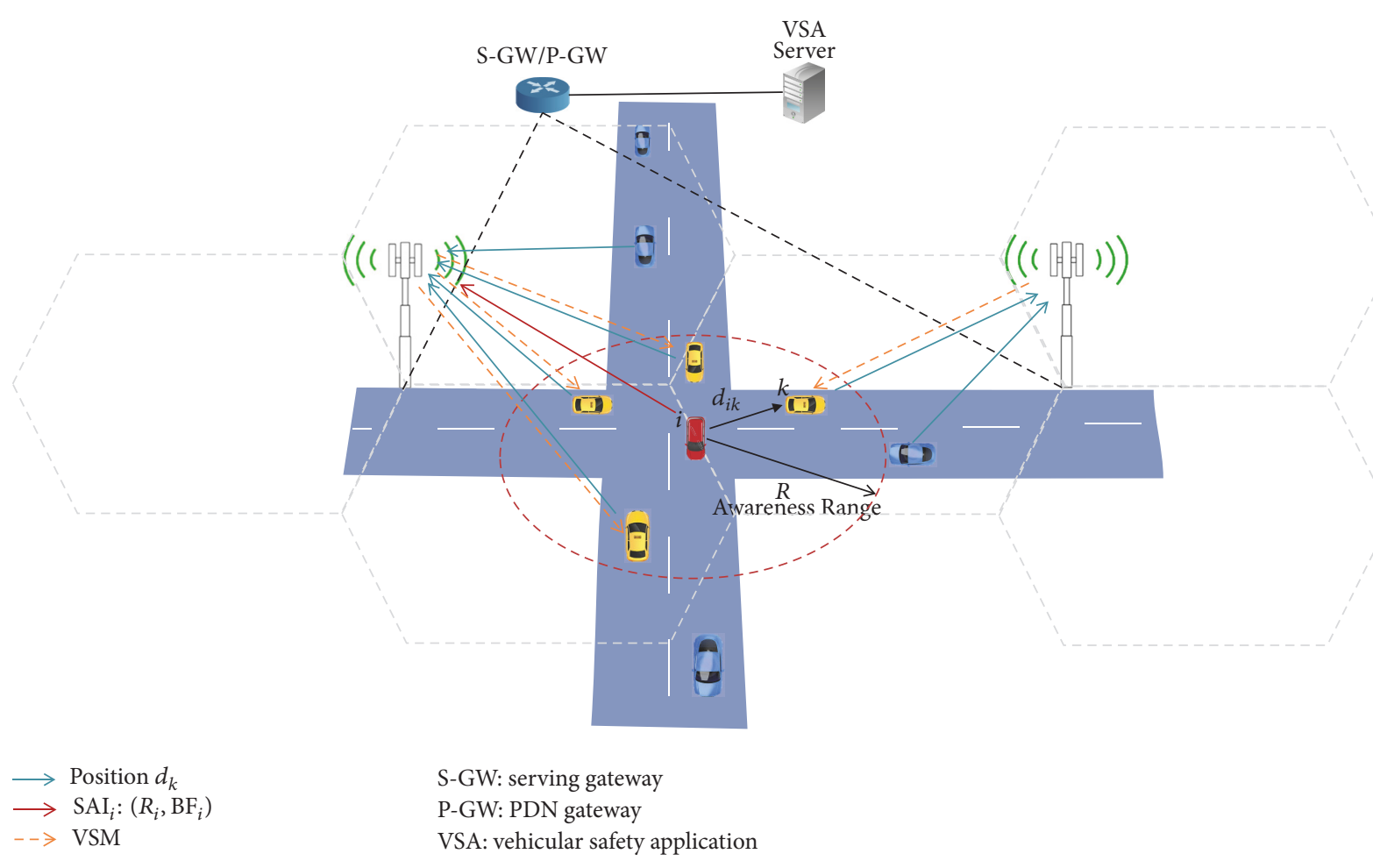

FIGURE 1: LTE multicell system and awareness range.

will also extract the SAI information from the received packet. This SAI information will then be checked against the database stored in the server (Table 2). With this SAI, the server retrieves the transmission beacon frequency $\mathrm{BF}_{i}$ and awareness range $R_{i}$ requirement of the application that vehicle $i$ is utilizing. Using the required awareness range, the server determines the forwarding set of vehicles (neighboring vehicles) by

$$
\mathbf{F}_{i}=\left\{\forall k: d_{i k}<R_{i}, i \neq k\right\},
$$

where $d_{i k}$ is the distance from vehicle $i$ to the neighboring vehicle $k$. Recalling the concept of data freshness defined by [15], the VSA server will discard the packet if it arrives later than the beacon inter arrival time $\left(1 / \mathrm{BF}_{i}\right)$.

2.2. MAC Layer Inclusion. This work proposes the inclusion of SAI from application layer to MAC Protocol Data Unit (PDU) control elements. This inclusion in MAC layer brings the processing towards the base station, decreasing latency and reducing the vehicular traffic load on the mobile network.

In cellular networks, eNB uses the cell radio network temporary identifier (C-RNTI) to uniquely identify UEs. As shown in Figure 3, a unique C-RNTI is assigned to every user by the eNB during the initial random access procedure which is used for identifying the radio resource control (RRC) connection and for scheduling purposes. Coding and decoding of physical downlink control channel (PDCCH) for a specific UE is based on its C-RNTI. UEs initiate contention based access to the network by transmitting a preamble sequence on the physical random access channel
(PRACH), to which the eNB responds with a C-RNTI on the physical downlink shared channel (PDSCH). In vehicular communications, since the awareness range is a function of vehicle's geographical location, if the transmitter's location is unknown to the network, an additional message containing transmitter's location is required.

So, for our proposed system model, signaling shown in Figure 3, the transmitter registers with the network in the same way as discussed above and it includes its location in the RRC connection request message, in its establishment cause as mo-Data, transmitted via the physical uplink shared channel (PUSCH). This location is introduced in the RRC connection request message as a new information element in line with the $3 \mathrm{GPP}$ specifications for RRC connection request [21]. At the same time, all the UEs already registered to the network update the eNB of their location using the network assisted global navigation satellite system (GNSS) location update message as specified in [35]. Once the vehicle has a beacon ready for transmission, it sends out a scheduling request (SR) in the physical uplink control channel (PUCCH). The eNB replies back in the downlink with the grant for sending BSR. After receiving this grant, the vehicle will send out the BSR in PUSCH containing the MAC PDU. SAI will be in the unused 16-bit long MAC Control Element inside the BSR. This element utilizes space currently reserved for future use and is indexed in the MAC PDU subheader by the logical channel ID (LCID) value equal to 01011 [36]. This new element, now referred to as SAI, is appended to the existing LCID values, such as common control channel (CCCH), C-RNTI, and the padding as shown in Figure 4. The eNB will then use the SAI to determine the 


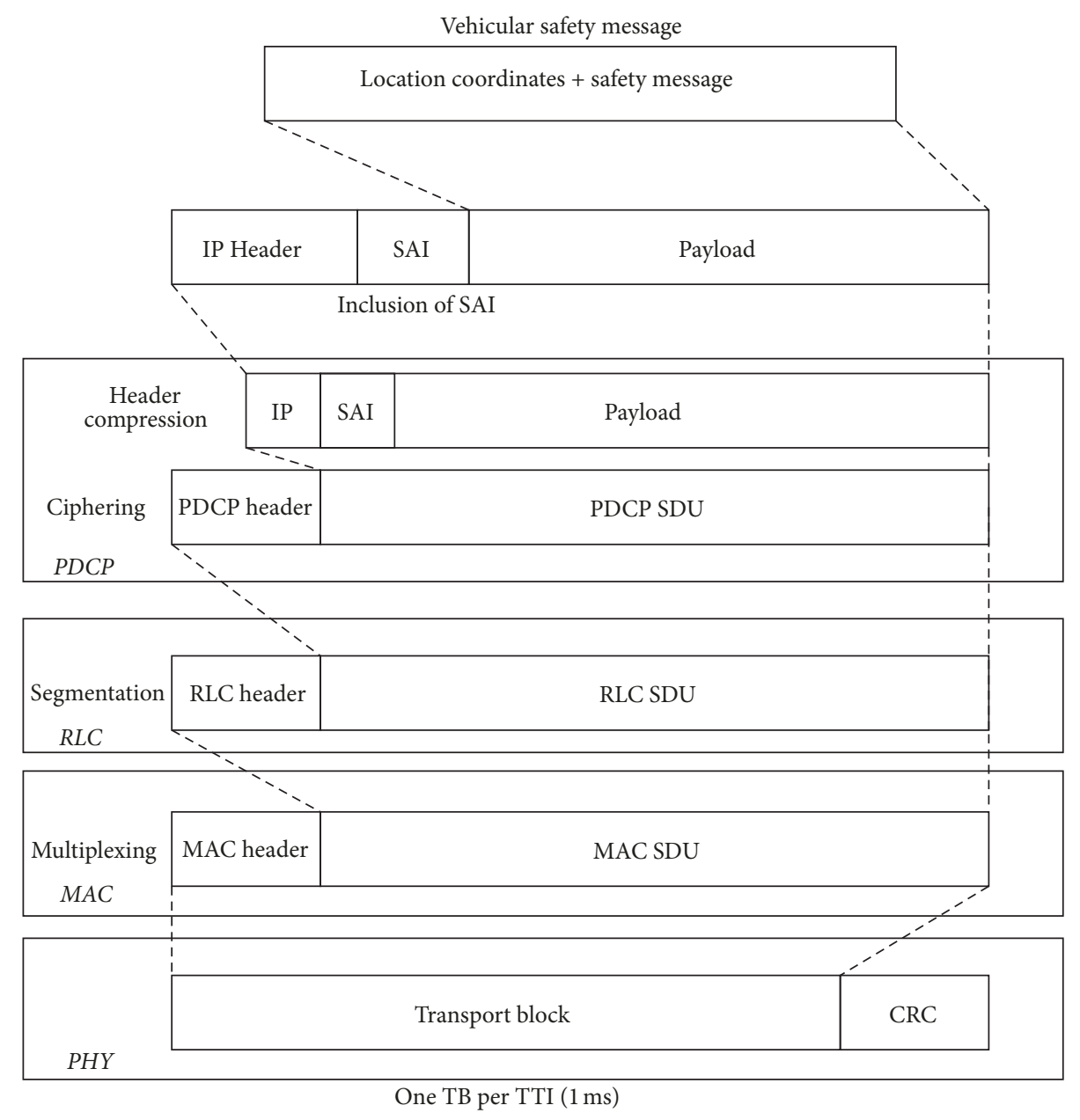

FIGURE 2: SAI inclusion in LTE message structure.

transmission parameters, allotting transmission resources to the sender and receiver vehicles.

2.3. Scalability of the System. Kato et al. [15] emphasized the significance of placing the VSA server close to the edge of LTE network. It can also be argued if a single server would be able to serve other locations, that is multiple eNodeBs or more servers would be required, giving rise to the question of system scalability. For the implementation of SAI algorithm at the VSA server, the location would play a vital role. Considering the data freshness concept, ideal location of the server would be at the EPC. Therefore, having multiple EPCs serving their respective geographical locations would have their own VSA servers with a similar approach as is for mobility management entity (MME), reducing the RTT while increasing the system capacity and eventually meeting the strict transmission requirements for vehicular safety applications.

In the case of having SAI appended in MAC layer, the implementation of SAI algorithm is brought towards the eNodeB, reducing the RTT and eventually increasing the system capacity. For vehicles being served by neighboring eNodeBs, the message would be forwarded by the eNodeB serving the source vehicle to the respective neighboring
eNodeB via the X2 interface. However, if the neighboring eNodeB does not have an X2 interface or is residing in a different EPC, then the VSA server would come into play. For such a scenario, the centralization of the server will again be significant.

\section{System Model}

The network is assumed to be composed of $N$ vehicles uniquely identified by their number $i,(i=1 \cdots N)$. Vehicles are assumed to use FDD LTE transceivers with $2 \times 10 \mathrm{MHz}$ bandwidth, uplink carrier frequency $1715 \mathrm{MHz}$ and downlink carrier frequency $2115 \mathrm{MHz}$ (band 4) [34, Table 5.5-1]. We refer to vehicles as LTE UEs.

The work in this paper builds on to the performance evaluation from [11] adding multicell and multipath along with Extended Vehicular A (EVA) fading environment. The network modeled is a $2 \times 2 \mathrm{~km}^{2}$ area of Manhattan Grid (MG) and Glasgow city center (GCC) shown in Figures 5 and 6, respectively, implemented in ns-3 [37]. Mobility of the vehicles in the network is generated using routes mobility model which assigns each vehicle with a route generated using Google maps API [14]. Along with the mobility model, the site configuration is also changed for 


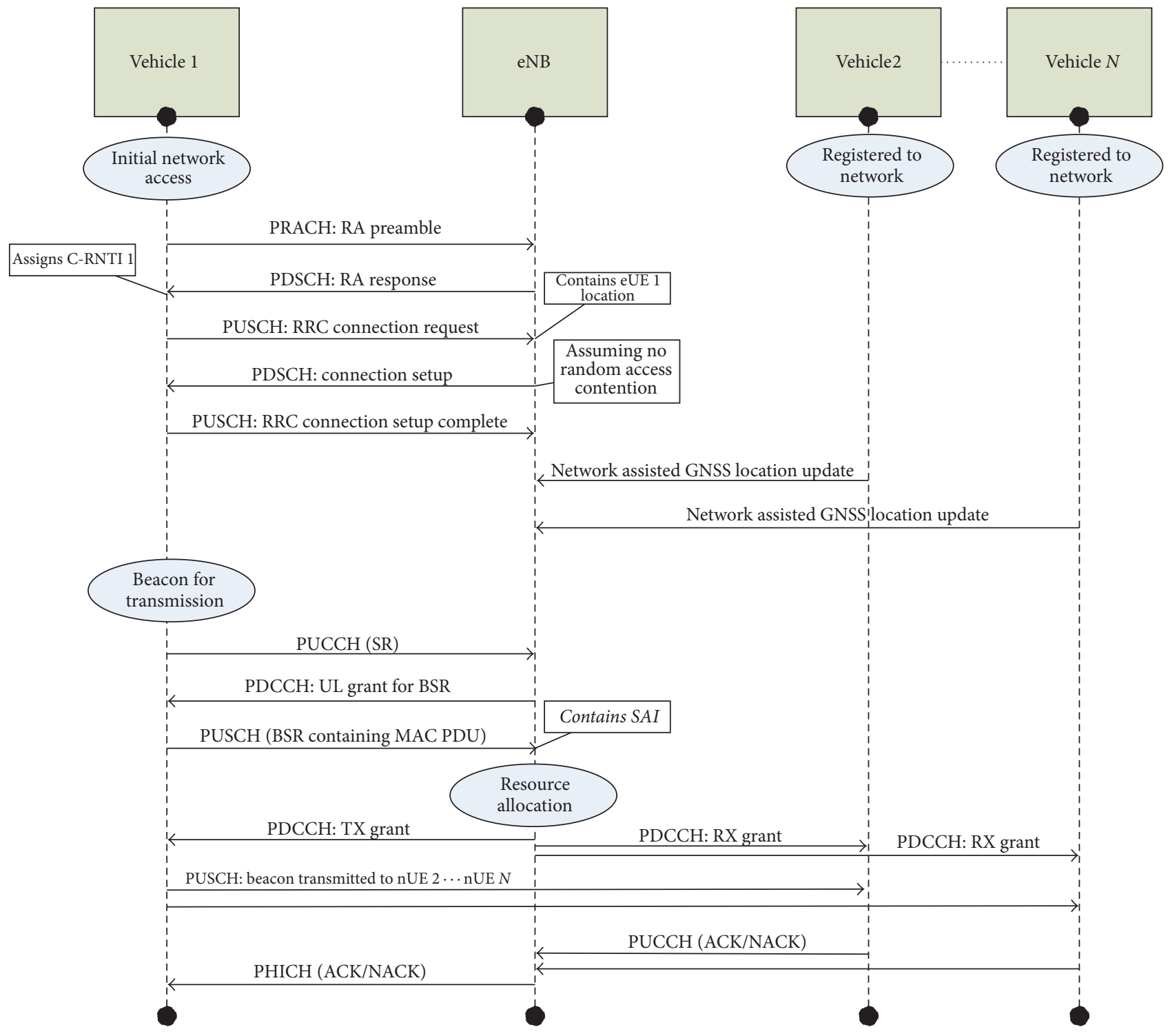

FIGURE 3: LTE signaling with MAC control elements in BSR.

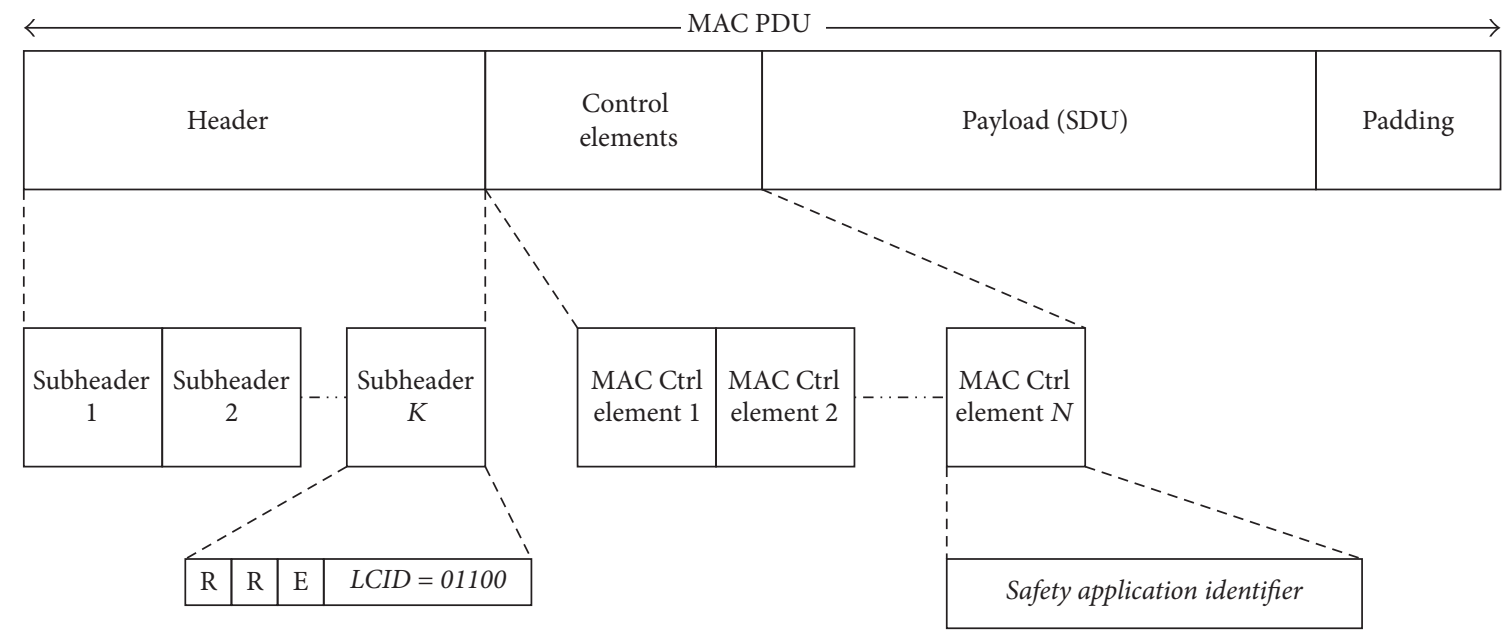

FIGURE 4: SAI in MAC control elements. 


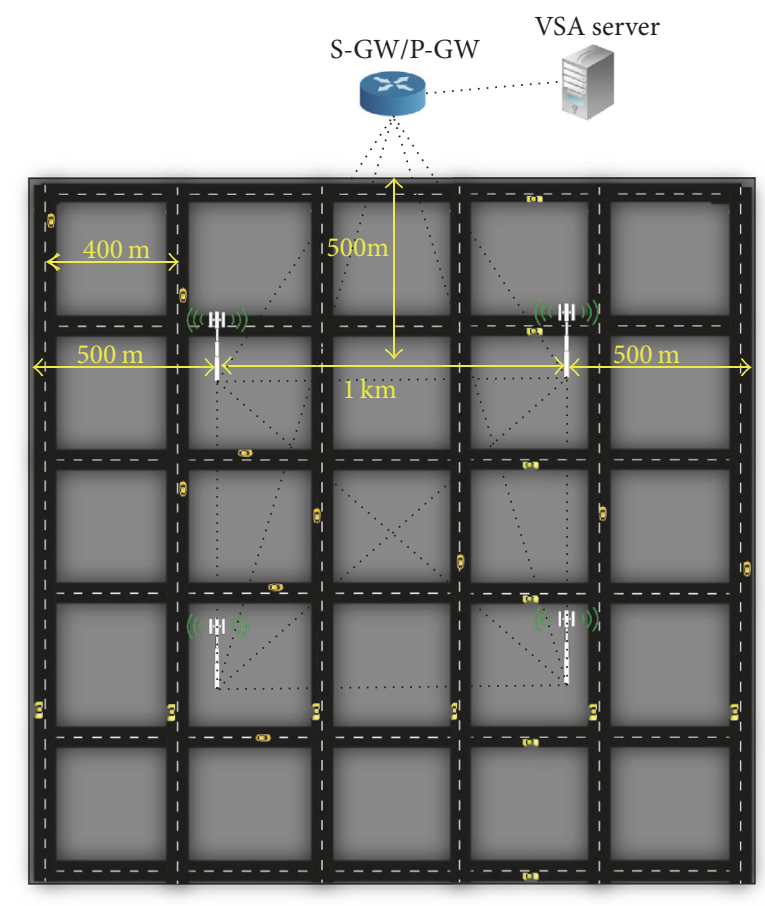

Figure 5: MG model covered by 4 sites and 3 cells/site with $1000 \mathrm{~m}$ intersite distance (ISD).

GCC. This change in the network design considers a more realistic network model employing the site configuration used by UK's mobile operator EE in Glasgow [8]. The LTE functionality is implemented through the LTE EPC network simulator (LENA) module [38] that includes highly detailed eNB and UE functionalities as specified by 3GPP standards. To manage handover and Internet connections, eNBs are interconnected through their X2 interfaces and connected to the EPC through S1-U interface. Interconnection from the PGW to the VSA server residing in the EPC is modeled using an error free 10 Gbps point-to-point link and TCP/IP version 4 .

The granularity of the LENA module is up to resource block (RB) level, including the user plane, control plane, and reference signals used for coherent reception. The safety messages are UDP packets and often might be segmented at the radio link control (RLC) and MAC layers to be transmitted on the transport shared channel ( $\mathrm{S}-\mathrm{CH})$ passed to the physical (PHY) layer. The size of transport blocks (TB) used at the PHY layer is decided by the MAC sublayer depending on the channel quality and scheduling decisions and may be transmitted using several RBs utilizing a modulation and coding scheme (MCS) [38]. Transmissions are scheduled every $1 \mathrm{~ms}$ corresponding to one subframe using Proportional Fair Algorithm, which is one of the approaches suitable for applications that include latency constraints [1, Chapter 12]. For downlink, the algorithm utilizes user's channel quality indicator (CQI $\in[0 \cdots 15]$ ) measurement reports sent from the vehicles. For uplink, channel quality measurements are done by the eNB through scheduling periodic vehicle transmissions of sounding reference signals (SRS). The radio resource control (RRC) module in LENA ns3 assigns the periodicity of SRS as a function of the number of UEs attached to an eNB according to 3GPP UE-specific procedure [37].

Each site, modeled by an eNB, uses $10 \mathrm{~W}(40 \mathrm{dBm})$ total transmission power and a cosine antenna sector model with $65^{\circ}$ half power beamwidth (HPBW) [39] and azimuthal direction $0^{\circ}, 120^{\circ}$ or $240^{\circ}$ with respect to north and $1 \mathrm{~m}$ intersector space. We assume utilization of $2 \times 10 \mathrm{MHz}$ bandwidth, which means there are $M=50 \mathrm{RBs}$ for the uplink and for the downlink. Therefore, assuming that the radio propagation channel is constant in subframe $t$, if vehicle $i$ is scheduled to receive from its serving cell $j$ on resource block $m \in[0 \cdots 49]$, the received signal power $P_{j i}$ can be modeled as follows:

$$
P_{j i}(m, t)=\frac{P_{j}(m, t) G_{j}\left(\theta_{j i}\right) G_{i}\left(\theta_{i j}\right)}{L_{j i}(m, t)},
$$

where $P_{j}(m, t)$ is the transmitted power for resource block $m$, $G_{j}\left(\theta_{j i}\right)$ is the antenna gain of cell $j$ in the direction of user $i$, $G_{i}\left(\theta_{i j}\right)$ is the antenna gain of user $i$ in the direction of cell $j$, and $L_{j i}$ is the path loss from cell $j$ to user $i$.

In the single cell scenario, there is no intercell interference and mutually exclusive RBs are assigned to users. However, in a more general deployed urban environment, the available spectrum is utilized by multiple cells in the service area and the intercell interference is one important limiting aspect, especially for cell-edge users [1, Chapter 12].

In order to allocate users' transmissions, while at the same time maximizing the signal to interference plus noise ratio (SINR) for each RB, frequency reuse, and handover are used. Frequency allocation has an impact on the achievable system capacity and delay since the transport block size, MCS, and the transmission schedule are dependent on the SINR [37, Chapter 9]. In ns-3, if several RBs are used to transmit a TB, the vector composed of the SINRs on each RB is used to evaluate the TB error probability. The SINR in RB $m$ when user $i$ is scheduled to receive from serving cell $j$ can be written as follows:

$$
\begin{aligned}
& \operatorname{SINR}_{i}(m, t) \\
& \qquad=\frac{P_{j}(m, t) G_{j}\left(\theta_{j i}\right) G_{i}\left(\theta_{i j}\right) / L_{j i}(m, t)}{N_{0}+\sum_{\forall l \neq j}\left(P_{l}(m, t) G_{l}\left(\theta_{l i}\right) G_{i}\left(\theta_{i l}\right) / L_{l i}(m, t)\right)},
\end{aligned}
$$

where $N_{0}$ is the noise power.

UEs report the SINR mapped to the CQI value corresponding to the MCS $(\in[0 \cdots 28])$ that ensures TB error rate $\leq 0.1$ according to the standard [40, Section 7.2.3]. In ns- 3 the downlink SINR is used to generate periodic wideband CQIs feedback (i.e., an average value of all RBs) and inband CQIs (i.e., a value for each RB). We set the CQI to be calculated by combining the received signal power of the reference signals to the interference power from the physical downlink shared channel as an approximation to (3).

For dynamic frequency allocation, we use the distributed fractional frequency reuse algorithm that utilizes intercell 


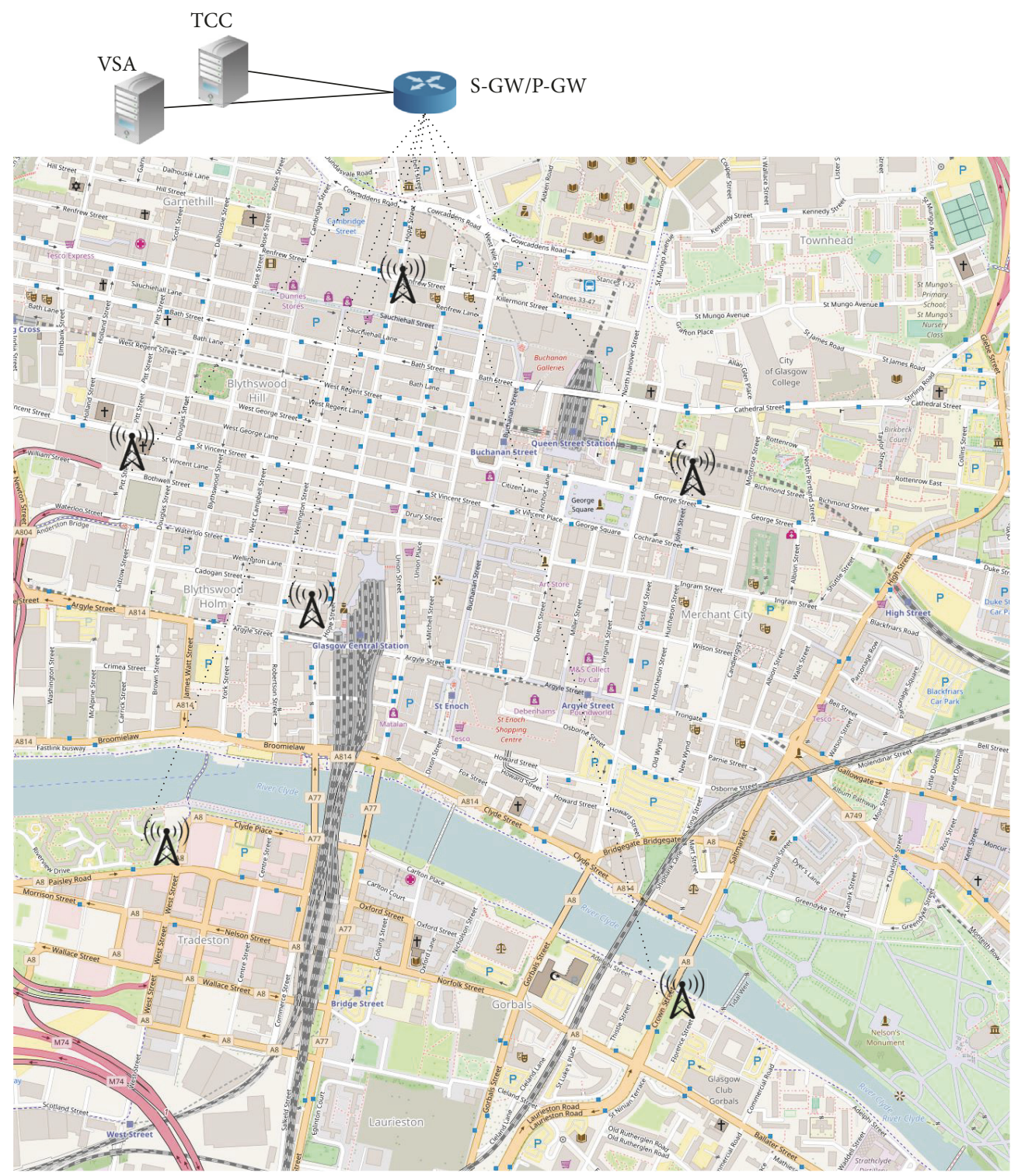

FIGURE 6: GCC model covered by 6 sites and 3 cells/site, where eNB location is determined using UK operator EE mast data [8].

interference coordination (ICIC) [41]. The eNB adaptively selects a set of RBs, referred to as cell-edge subband (set to $10 \mathrm{RBs}$ ), based on information exchanged with its neighbors. Therefore, according to ICIC, a vehicle should be allocated in the cell-edge subband if its serving cell reference signal received quality (RSRQ) gets lower than $-10 \mathrm{~dB}$.

Handover decisions are also performed by eNBs based on configurable event-triggered measurement reports, including the RSRQ and the reference signal received power (RSRP). The measurements are performed by UEs usually in orthogonal frequency division multiplexing (OFDM) symbols carrying reference signals (RS) for antenna port 0 . In ns3 , since the channel is assumed flat over an $R B$ that is composed of 12 subcarriers, all resource elements (RE) in an RB have the same power. Hence, assuming orthogonal RS reception, the RSRP for cell $j$ measured by vehicle $i$ is given by

$$
\begin{aligned}
\operatorname{RSRP}_{j i}(t) & =\frac{\sum_{m=0}^{M-1} \sum_{k=0}^{K-1}\left(P_{j i}(m, k, t) / K\right)}{M} \\
& =\frac{\sum_{m=0}^{M-1}\left(P_{j i}(m, t) / 12\right)}{M},
\end{aligned}
$$

where $P_{j i}(m, k, t)$ is the received power of RS $k$ in RB $m, M$ is the number of RBs, and $K$ is the number of RS measured in the RB. The average value is passed to higher layers every $200 \mathrm{~ms}$; therefore the index $t$ is omitted for simplicity.

The RSRQ for serving cell $j$ measured by vehicle $i$ can be computed by the following [42]:

$$
\operatorname{RSRQ}_{j i}=\frac{M \times \operatorname{RSRP}_{j i}}{\operatorname{RSSI}_{j i}},
$$

where $\operatorname{RSSI}_{j i}$ is the received signal strength indicator (RSSI) measured by UE $i$ when served by cell $j$ and, according to the 
TABLE 3: LTE simulation parameters.

\begin{tabular}{|c|c|}
\hline Parameter & Value \\
\hline Simulation time & 100 seconds \\
\hline Road model & $\begin{array}{c}\text { Manhattan grid model (MG) (two way roads) } \\
\text { Glasgow City Center (GCC) }(2 \mathrm{~km} \times 2 \mathrm{~km})\end{array}$ \\
\hline LTE network & $\begin{array}{l}4 \text { sites with } 3 \text { cells/site, } 1000 \mathrm{~m} \text { ISD for MG } \\
6 \text { sites with } 3 \text { cells/site, UK Operator EE mast data [8] }\end{array}$ \\
\hline Transmission power & eNB: $40 \mathrm{dBm}, \mathrm{UE}: 23 \mathrm{dBm}$ \\
\hline Carrier frequency DL/UL & $2115 \mathrm{MHz} / 1715 \mathrm{MHz}$ \\
\hline Channel bandwidth & $2 \times 10 \mathrm{MHz}(2 \times 50 \mathrm{RBs})$ \\
\hline Noise Figure & eNB: $5 \mathrm{~dB}, \mathrm{UE}: 9 \mathrm{~dB}$ \\
\hline UE antenna model & Isotropic $(0 \mathrm{dBi})$ \\
\hline eNB antenna model & $15 \mathrm{~dB}$ Cosine model, $65^{\circ} \mathrm{HPBW}$ \\
\hline Scheduling algorithm & Proportional Fair \\
\hline Handover algorithm & $\begin{array}{l}\text { A2A4RSRQ, RSRQ threshold }-5 \mathrm{~dB} \text {, } \\
\text { and NeighbourCellOffset }=2(1 \mathrm{~dB})\end{array}$ \\
\hline Frequency reuse & Distributed Fractional Freq. Reuse \\
\hline \multirow{2}{*}{ Path loss model } & LogDistance $(\alpha=3)$ and \\
\hline & 3GPP Extended Vehicular A model \\
\hline Safety message format & 256 bytes UDP \\
\hline Number of vehicles & $75,100,125,150$ \\
\hline Average vehicle's speed & $20 \mathrm{~km} / \mathrm{h}, 40 \mathrm{~km} / \mathrm{h}$ \\
\hline Beacon frequency & $1 \mathrm{~Hz}, 10 \mathrm{~Hz}$ \\
\hline Awareness range $(R)$ & $100 \mathrm{~m}, 250 \mathrm{~m}, 500 \mathrm{~m}, 750 \mathrm{~m}, 1000 \mathrm{~m}$ \\
\hline
\end{tabular}

standard, comprises the linear average of the total received power observed by the UE from all sources, including cochannel serving and nonserving cells, adjacent channel interference, thermal noise, and so forth [42]. The RSSI measurement model in ns-3 is computed considering two RE per RB (the one that carries the RS) [37], that is,

$$
\begin{aligned}
& \operatorname{RSSI}_{j i}(t) \\
& \quad=\sum_{m=0}^{M-1} 2 \times\left(\frac{N_{0}}{12}+\sum_{\forall l} \frac{P_{l}(m, t) G_{l}\left(\theta_{l i}\right) G_{i}\left(\theta_{i l}\right)}{12 \times L_{l i}(m, t)}\right) .
\end{aligned}
$$

Furthermore, in order to perform handover decisions, the reported RSRQ for neighboring cell $l$, when vehicle $i$ is connected through serving cell $j$, is computed by the following:

$$
\mathrm{RSRQ}_{l i}=\frac{M \times \mathrm{RSRP}_{l i}}{\mathrm{RSSI}_{j i}} .
$$

The RSRQs for serving and neighboring cells are used with the A2A4RsrqHandoverAlgorithm to trigger LTE events A2 and A4 [43, Section 5.5.4]. Event A2 is set to be triggered when the RSRQ of the serving cell becomes worse than $-5 \mathrm{~dB}$ (ServingCellThreshold parameter). While event A2 is true, event A4 is triggered if a neighboring cell becomes better than a threshold, which is set by the algorithm to a very low value for the trigger criteria to be true. Thus, the measurement reports received by the eNB are used to consider a neighboring cell as a target cell for handover only if its RSRQ is $1 \mathrm{~dB}$ higher than the serving cell (NeighbourCellOffset parameter). Table 3 summarizes the simulation parameters for LTE.

3.1. Vehicular Radio Urban Environment. As mentioned in Section 1.1, previous evaluations lack the use of multipath and multicell environments. Use of proper channel modeling is essential in evaluating system models as it poses close to reality system degradations. The system model presented in this paper consists of 6 sites with 3 cells per site. Users are assumed to have isotropic antenna $\left(G_{i}\left(\theta_{i j}\right)=0 \mathrm{dBi}\right)$ and the path loss $\left(L_{j i}\right)$ is modeled using the Log-distance propagation with shadow exponent, $n=3$ [44] plus 3GPP EVA multipath fading model, $\mathscr{L}_{j i}$ [34], and is computed by the following:

$$
L_{j i}(m, t)=L_{0}+10 n \log _{10}\left(\frac{d_{j i}(t)}{d_{0}}\right)+\mathscr{L}_{j i}(m, t) \mathrm{dB},
$$

where $L_{0}=20 \log _{10}\left(\lambda /\left(4 \pi d_{0}\right)\right), \lambda$ is the wavelength, and $d_{0}$ is the reference distance assumed to be $10 \mathrm{~m}$ in our simulations. Traces for EVA were generated using MATLAB while modifying the classical Doppler spectrum for each tap at vehicular speed $(v)$ of $40 \mathrm{~km} / \mathrm{h}$ and $60 \mathrm{~km} / \mathrm{h}$ as

$$
S(f) \propto \frac{1}{\left(1-\left(f / f_{D}\right)^{2}\right)^{0.5}},
$$

where $f_{D}=v / \lambda$. These EVA traces specified by 3GPP in [34] improvise the NLOS conditions relative to the speed 
of UE and the transmission bandwidth. Simulation of the multipath fading component in ns-3 uses the trace starting at a random point along the time domain for a given user and serving cell. The channel object created with the rayleighchan function is used for filtering a discrete-time impulse signal in order to obtain the channel impulse response. The filtering is repeated for different Transmission Time Interval (TTI), thus yielding subsequent time-correlated channel responses (one per TTI). This channel response is then processed with the pwelch function for obtaining its power spectral density values, which are then saved in a file with the format compatible with the simulator model.

3.2. SAI Implementation Model. Archer and Vogel in [45] carried out a survey on traffic safety problems in urban areas. It was concluded that overtaking tends to occur less frequently within urban areas where the speed limit is less than $50 \mathrm{~km} / \mathrm{h}$. Lane changing, however, occurs quite frequently within urban areas due to low speed limits. The number of rear-end accidents is greater within urban areas than in rural areas and similarly, due to higher level of congestion and higher number of traffic junctions, there is a greater number of opportunities for turning and crossing accidents within urban area. According to another survey of 4500 crashes by the Insurance Institute of Highway Safety [46], $13 \%$ of accidents are during lane change maneuvers, $12 \%$ are intersection collisions, $22 \%$ are due to traffic light violation, $9 \%$ are head on collisions, $18 \%$ include left turning crashes, $12 \%$ are due to emergency vehicles parked on blind turns, and $14 \%$ are due to over speeding. In the light of these statistics, for our adopted urban environment, the SAIs (Table 2) we utilize in our simulations are $1(25 \%), 3(22 \%), 5$ (9\%), 6 (18\%), 7 (12\%), and $9(14 \%)$.

3.3. Background Traffic. In order to model close to real scenarios, we added background traffic into our simulations. Modeling of such traffic is done by making $50 \%$ of the existing vehicles use voice application and $25 \%$ stream videos. Voice application is modeled by implementing constant bit rate (CBR) traffic generator on G.711 codec using onoffapplication on ns-3 [37]. Voice payload is 172 bytes and the data rate chosen is $68.8 \mathrm{Kbps}$. The reason for using a high data rate is to cater worst case scenario where an operator would prefer having a high quality voice application. At the same time, video streaming is being carried out using evalvid simulator module on $\mathrm{H} .263$ codec with a payload of 1460 bytes at a data rate of 1.22 Mbps [47]. Both these voice and video applications are assigned their respective QCIs.

3.4. Performance Measures. The primary performance measures used are the end-to-end packet delay, the packet delivery ratio, and the downlink goodput. The goodput is defined as the number of useful information bits received at the application layer per unit of time.

In addition, we use the notation $\overline{\left|\mathbf{F}_{i}\right|}$ to represent the average number of neighbors, calculated over the number of receivers whenever a beacon is disseminated. The end-to-end delay of a beacon $\left(D_{i k}\right)$ is defined as the time measured from the start of its transmission at vehicle $i$ 's application layer until its successful reception at vehicle $k$ 's application layer. PDR is defined as the beacon success rate within the awareness range $R$ [48]; that is, for a beacon sent from vehicle $i$, its PDR is given by

$$
P_{i}(R)=\sum_{k \in \mathbf{F}_{i}} \frac{I_{i k}}{\left|\mathbf{F}_{i}\right|} ; \quad\left|\mathbf{F}_{i}\right|>0,
$$

where $|\cdot|$ indicates the cardinality of the set and $I_{i k}(\in\{0,1\})$ is set to 1 if the beacon is received by vehicle $k$.

\section{Simulation Results}

The success of cellular networks relies on the scalability of the system capacity achieved by reusing the spectrum. The common approach implemented by operators is to cater the capacity needs as the traffic demand grows. Therefore, first we analyze the capacity limit which results from the single cell case compared to multiple cells using the Friis radio propagation environment and afterwards we analyze the impact of the urban radio environment as a function of the awareness range.

Figure 7 (a) shows the cumulative distribution function (CDF) of the end-to-end delay when the network is composed of 100 vehicles moving at an average speed of $40 \mathrm{~km} / \mathrm{h}$. The service area is covered using a single cell with an isotropic antenna located at the upper left corner of Manhattan Grid model as utilized by [11] in a Friis radio environment where vehicles transmit at $1 \mathrm{~Hz}$ beacon frequency. We observe that, for awareness range of up to $500 \mathrm{~m}$, the end-to-end delay is $\leq 100 \mathrm{~ms}$ with around $95 \%$ probability. Probability of higher end-to-end delay increases more between $250 \mathrm{~m}$ to $500 \mathrm{~m}$ as compared to the increase from $500 \mathrm{~m}$ to $750 \mathrm{~m}$. That is mainly the result of the increase in traffic, but also as the awareness range increases, cars located on roads near the edge of the Manhattan model will have neighbors towards the cell direction with better signal quality, which is further exploited by the scheduling algorithm. Figure 7 (a) also shows that there is no significant increase in end-to-end delay from $750 \mathrm{~m}$ to $1000 \mathrm{~m}$. This is due to the border effect, incurred by the car located at the edge of the model having less number of neighbors when increasing the awareness range as compared to the cars located at the center of the cell. Nevertheless, these results show that up to 100 vehicles were possible for the single cell case at a beacon frequency of $1 \mathrm{~Hz}$ under Friis radio environment. In order to analyze the intercell interference impact on the delay while increasing the number of vehicles and beacon frequency, we increase the network capacity by deploying 4 sites with 3 sectors/site (the average number of vehicles per sector is reduced to 12.5). Figure 7(b) shows that the end-to-end delay is then further improved to below $50 \mathrm{~ms}$, with $91 \%$ success rate when awareness range of $1000 \mathrm{~m}$ at the beacon frequency of $1 \mathrm{~Hz}$ was utilized. We can also notice that in this case there are some beacons that experienced longer delays compared to the single cell scenario caused by low quality conditions of cell-edge users due to intercell interference and handover. However, with $1000 \mathrm{~m}, 93.4 \%$ of the forwarded beacons were received with end-to-end delay $\leq 100 \mathrm{~ms}$ and $98.2 \%$ for $250 \mathrm{~m}$. 


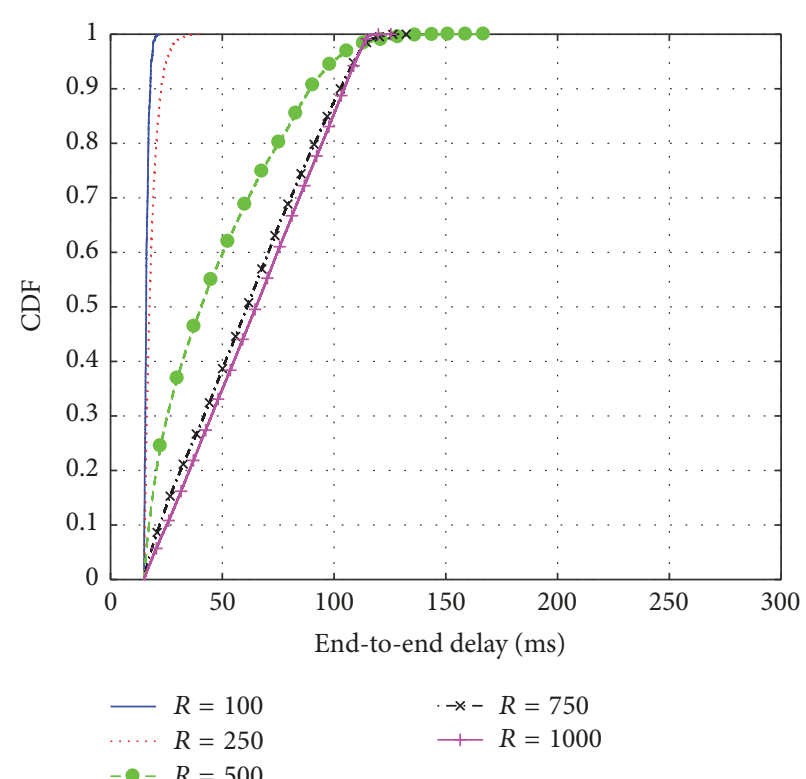

(a)

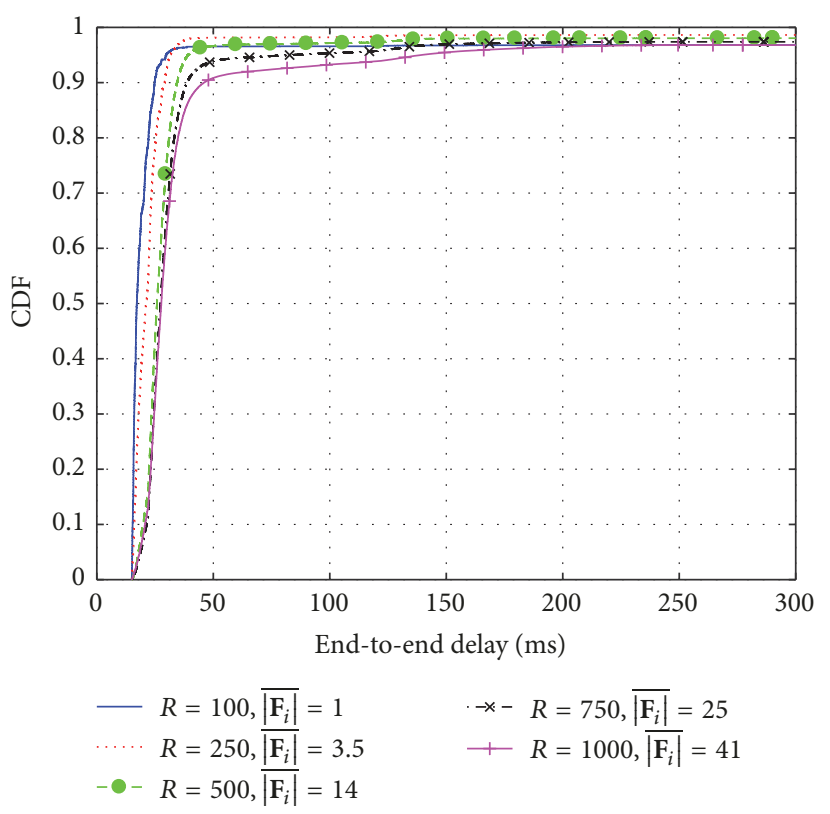

(b)

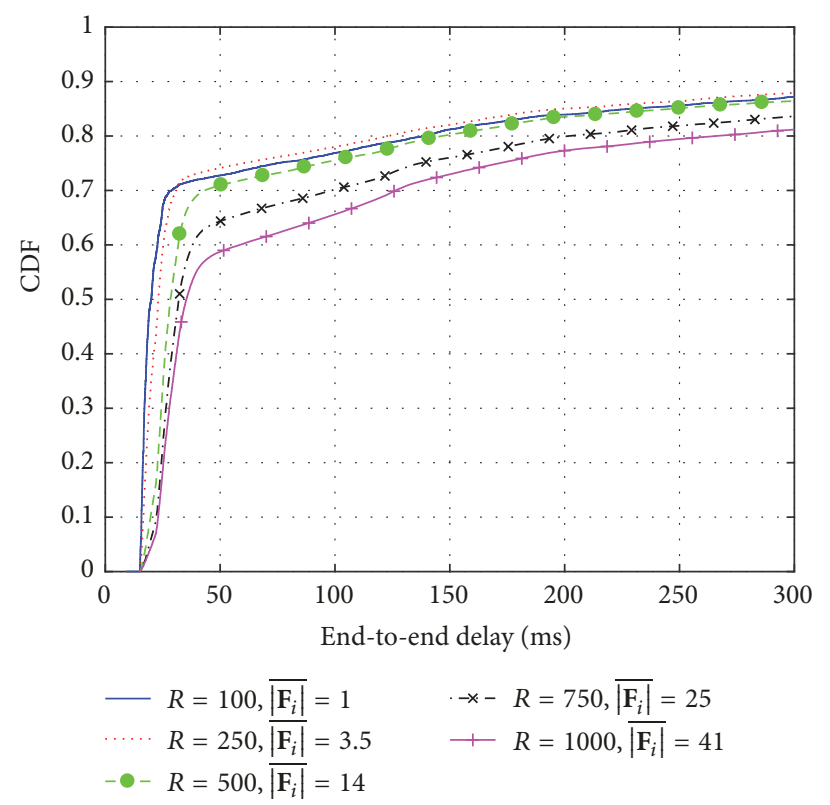

(c)

Figure 7: LTE end-to-end delay for 100 vehicles at a beacon frequency $1 \mathrm{~Hz}$ and average speed $40 \mathrm{~km} / \mathrm{h}$ with Friis radio environment: (a) single cell; (b) multicell (4 sites, 3 sectors/site); (c) multicell with EVA fading radio environment.

The results in Figure 7(b) show that, under the Friis radio propagation environment, the capacity increases and the system performance is not significantly affected in terms of the end-to-end delay in presence of intercell interference and handover since the scheduler effectively managed interference by time and frequency allocations. However, in an urban environment, it is expected that fading caused by buildings and Doppler shift due to the relative velocity of vehicles have a very important impact on the received signal quality [49]. Figure 7(c) shows the results when the radio propagation environment was changed to Log-distance-dependent and
3GPP EVA under the same site configurations. Under this radio environment the probability for the end-to-end delay to be less than or equal to $100 \mathrm{~ms}$ was around $76 \%-78 \%$ for awareness range between $100 \mathrm{~m}$ and $500 \mathrm{~m}, 70 \%$ for $750 \mathrm{~m}$, and $66 \%$ for $1000 \mathrm{~m}$. Hence, the significant effect on the endto-end delay is because of path loss and frequency selective fading imposed by the EVA fading environment. The end-toend delay for beacons to and from cell-edge users significantly increases. Similarly, for inner cell users, the end-to-end delay remained close to the Friis results and for up to $500 \mathrm{~m}$ the end-to-end delay was $\leq 50 \mathrm{~ms}$ with $74 \%$ to $71 \%$ probability, 


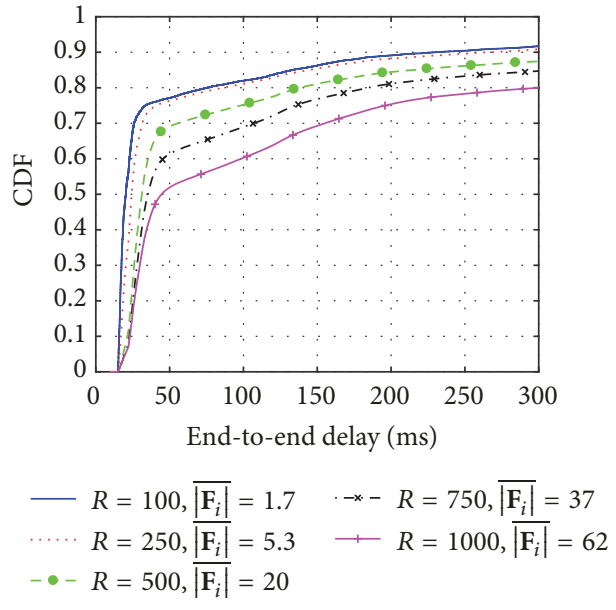

(a)

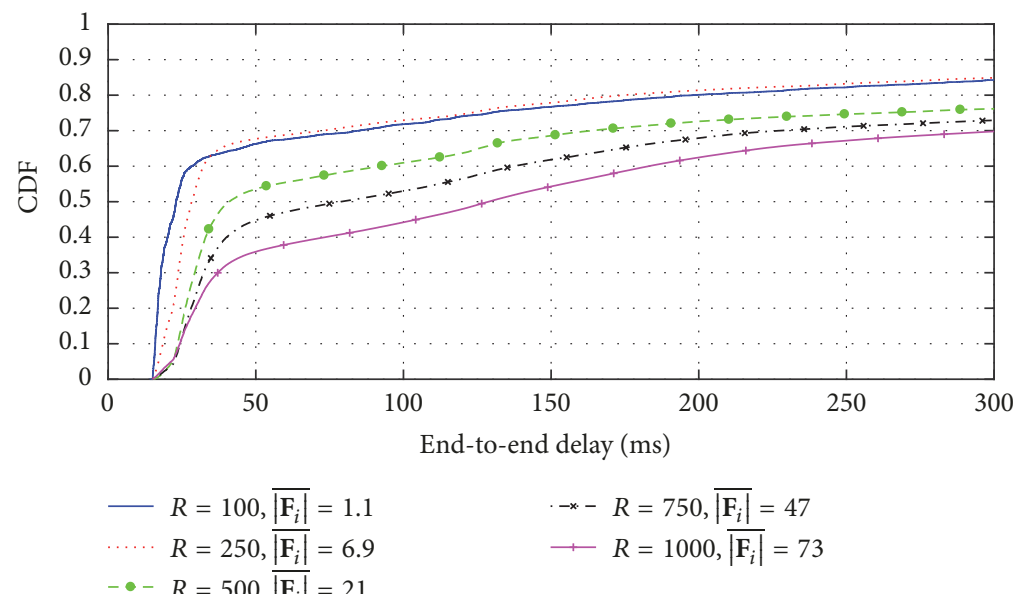

(b)

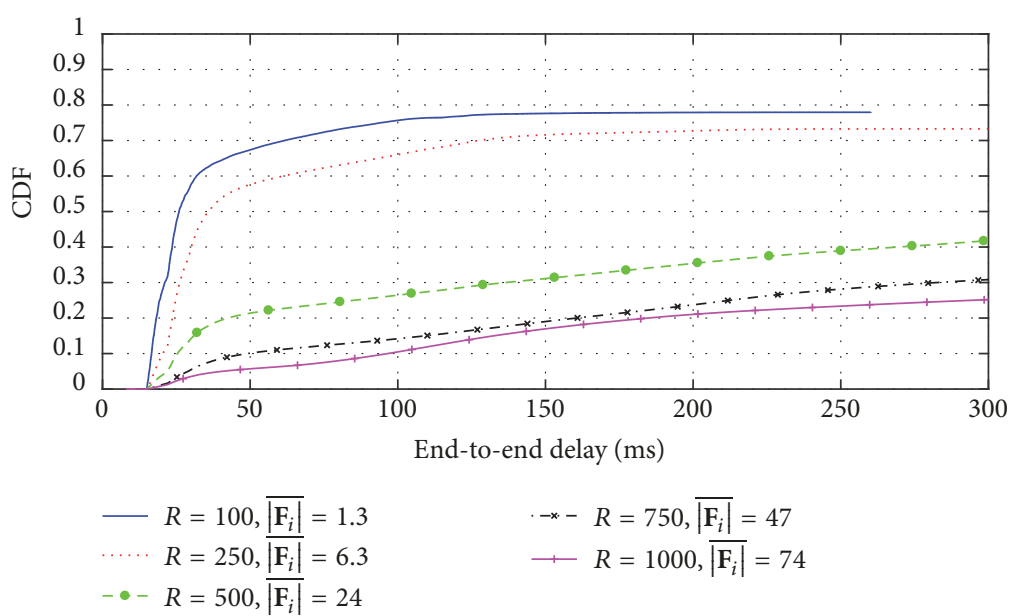

(c)

FIGURE 8: End-to-end delay for sample networks at average speed $40 \mathrm{~km} / \mathrm{h}$ with 150 vehicles at beacon frequency: (a) $1 \mathrm{~Hz}$ with MG model; (b) $1 \mathrm{~Hz}$ with GCC model; (c) $10 \mathrm{~Hz}$ with GCC model.

$65 \%$ for $750 \mathrm{~m}$, and $59 \%$ for $1000 \mathrm{~m}$. Therefore, it can be deduced that, with the addition of multipath fading along with critical radio propagation environment, probability of delay being $\leq 50 \mathrm{~ms}$ decreases by $19 \%$ to $30 \%$.

Continuing on with multicell and EVA fading radio environment, by increasing the network size, the number of vehicles in the awareness range increases and the end-to-end delay increases as shown in Figure 8(a). Nevertheless, the end-to-end delay remained below $100 \mathrm{~ms}$ for up to $250 \mathrm{~m}$ with $82 \%$ probability for 100 vehicles and similar rate was obtained with up to 150 vehicles. When the awareness range increases to $500 \mathrm{~m}$ the probability was $80 \%$ for 100 vehicles while for 150 vehicles further reduces to $73 \%$. For awareness range of $750 \mathrm{~m}$ and $1000 \mathrm{~m}$ the probability reduced to $75 \%$ and $71 \%$ for 100 vehicles while for 150 vehicles reduces to $69 \%$ and $60 \%$, respectively.

Changing the simulation model along with mobility traces to Glasgow city center, the effect on delay performance with 150 vehicles at $1 \mathrm{~Hz}$ and $10 \mathrm{~Hz}$ is shown in Figures 8(b) and $8(\mathrm{c})$, respectively. It is interesting to observe the effect of changing the mobility model to a European city. Where each city block is $400 \mathrm{~m}$ in the MG model, average city block size in GCC is around $200 \mathrm{~m}$, increasing vehicle density, eventually growing the average number of neighbors for GCC model. This can be observed for an awareness range of $250 \mathrm{~m}$, the probability of delay to be $\leq 50 \mathrm{~ms}$ decreases from $77 \%$ to $68 \%$ while for $1000 \mathrm{~m}$ this deterioration goes from $54 \%$ to $37 \%$, hence, an overall decrease of about $9 \%$ to $17 \%$ for various awareness ranges. Similarly, in Figure 8(c), it is observed that changing the beacon frequency from $1 \mathrm{~Hz}$ to $10 \mathrm{~Hz}$ with an awareness range of $500 \mathrm{~m}$ the probability of delay to be $\leq 50 \mathrm{~ms}$ drastically decreases from $55 \%$ to $22 \%$. Whereas, for an awareness range of $1000 \mathrm{~m}$, degradation of about $29 \%$ is observed. Performance with a high beacon frequency such as $10 \mathrm{~Hz}$ is overall seen to be poor. That is because packets that arrive late are discarded and are mainly sent by cell-edge users for which their neighborhood very likely includes users at the cell edge. Hence, the delay from the uplink is indirectly used as a spatial filtering of low quality cell-edge users. This suggests that if the information is not useful when received after $100 \mathrm{~ms}$, the better use of resources is to drop the packet at the minimum latency requirement by the server. 


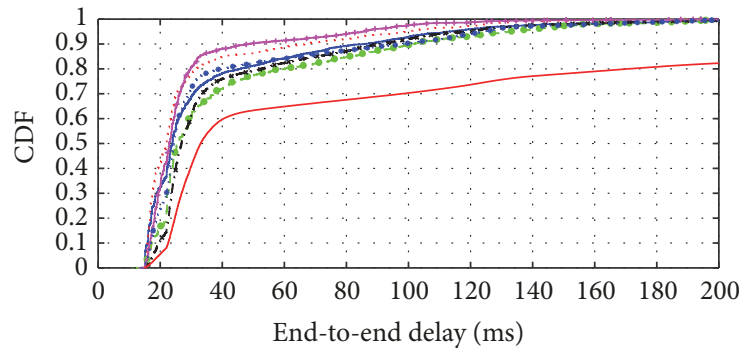

$$
\begin{aligned}
& -\mathrm{SAI}=1, \overline{\left|\mathrm{F}_{i}\right|}=1.9 \longrightarrow \mathrm{SAI}=7, \overline{\left|\mathrm{F}_{i}\right|}=5.6 \\
& \mathrm{SAI}=3, \overline{\left|\mathrm{F}_{i}\right|}=1.6 \quad \cdots \cdot \mathrm{SAI}=9, \overline{\left|\mathrm{F}_{i}\right|}=5.4 \\
& \text { - } \mathrm{SAI}=5, \overline{\left|\mathrm{F}_{i}\right|}=4.1 \quad \text { Without SAI, } \overline{\left|\mathrm{F}_{i}\right|}=16 \\
& - \text { - }-\mathrm{SAI}=6, \overline{\left|\mathrm{F}_{i}\right|}=14
\end{aligned}
$$

(a)

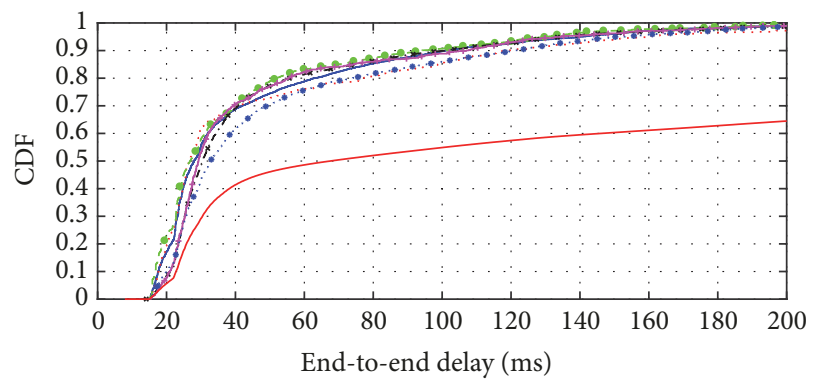

$\begin{aligned}-\mathrm{SAI} & =1, \overline{\left|\mathrm{F}_{i}\right|}=2.6 \\ \cdots \mathrm{SAI} & =3, \overline{\left|\mathbf{F}_{i}\right|}=2 \\ -\mathrm{SAI} & =5, \overline{\left|\mathrm{F}_{i}\right|}=3.5 \\ -\mathrm{S}-\mathrm{SAI} & =6, \overline{\left|\mathbf{F}_{i}\right|}=22\end{aligned}$

$\longrightarrow \mathrm{SAI}=7, \overline{\left|\mathrm{F}_{i}\right|}=7.8$

… SAI $=9, \mid \overline{F_{i} \mid}=9.4$

Without SAI, $\overline{\left|\mathrm{F}_{i}\right|}=21$

(b)

FIGURE 9: LTE end-to-end delay at average speed $40 \mathrm{~km} / \mathrm{h}$ (GCC) and (a) 100 vehicles and (b) 150 vehicles.

Next, we observe and compare the results after the proposed algorithm implementation. Figure 9 shows the CDF of the end-to-end delay for 100 and 150 vehicles at an average speed of $40 \mathrm{~km} / \mathrm{h}$ with and without the implementation of SAI algorithm. The scenario being compared has the awareness range set to $500 \mathrm{~m}$ and beacon frequency to $10 \mathrm{~Hz}$. We observe a significant decrease in the end-toend delay after SAI algorithm implementation. Probability for the end-to-end delay to be less than $100 \mathrm{~ms}$ is above $80 \%$ in both scenarios whereas, without the algorithm, this probability is below $70 \%$ for 100 vehicles (Figure 9(a)) and below $60 \%$ for 150 vehicles (Figure 9(b)). The variation in this delay between various SAIs is because of the difference in transmission parameters. Larger awareness range leading to higher number of neighbors $\mid \overline{F_{i} \mid}$ and higher beacon frequency results in more congestion and large queuing times. The reason for better delay values with SAI algorithm is mainly the result from restricting resources to the required transmission parameters for safety applications mentioned in Section 3.2.

Figure 10 shows the aggregate downlink goodput for the same scenario investigated for end-to-end delay. After the implementation of SAI algorithm, the downlink goodput for 100 vehicles dropped from 7.46 Mbps to 6.22 Mbps and for 150 vehicles it dropped from $21.67 \mathrm{Mbps}$ to $13.76 \mathrm{Mbps}$. This significant decrease in the downlink direction is again due to the restriction of unnecessary data dissemination from the VSA server to vehicles. Eventually, this decrease of goodput results in less load on the LTE system. The increase in the downlink goodput for the scenario without SAI explains the high end-to-end delay observed in Figure 9, showing a burst of packet in the downlink leading to waiting time in the queue.

Furthermore, the impact of regular cellular traffic on our LTE vehicular network is studied in order to validate our findings and algorithm. A broad picture of our observations under different scenarios is shown in Figure 11 where probability of end-to-end delay being less than or equal to $50 \mathrm{~ms}$ is studied. As expected, this probability decreases by about $3-4 \%$ when background traffic is added. However, with

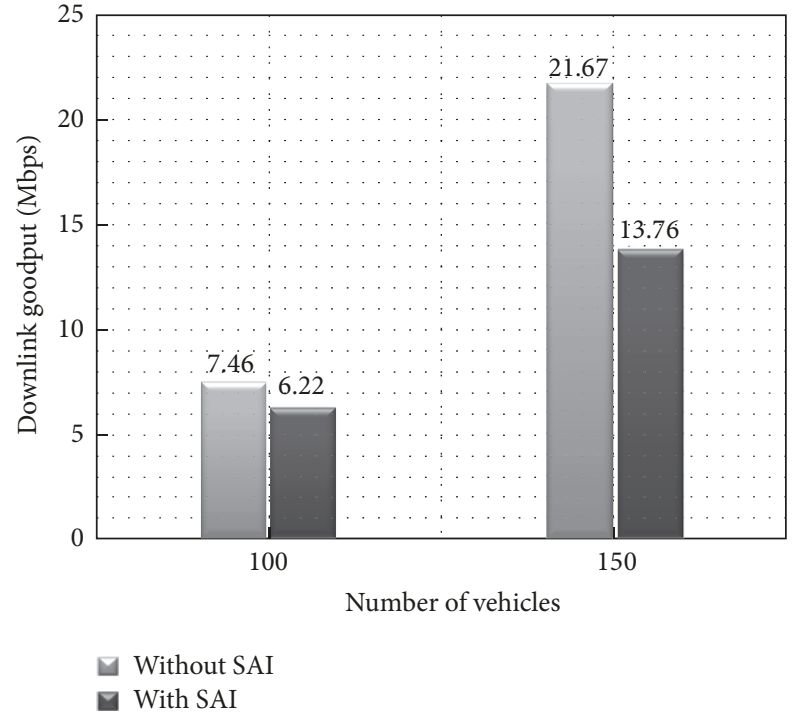

Figure 10: Downlink goodput for 100 and 150 vehicles at an average speed $40 \mathrm{~km} / \mathrm{h}$ (GCC).

SAI algorithm, probability of end-to-end delay being less than $50 \mathrm{~ms}$ stays above $90 \%$ even after adding background traffic.

Finally we include the SAI into MAC layer PDU control elements to prove our concept of D2D like unicast transmission between sender and receivers. Modeling of the system is carried out in accordance with the description in Section 2.2. Figure 12 shows end-to-end delay for 100 and 150 vehicles with and without the inclusion of SAI in MAC layer. It is evident that 150 vehicles experience almost the same probability for end-to-end delay being less than $50 \mathrm{~ms}$ as experienced by 100 vehicles. This shows that with the use of MAC layer control elements around 50\% more vehicles can be accommodated by LTE network, increasing the spectrum efficiency eventually leading to higher capacity of the system. 


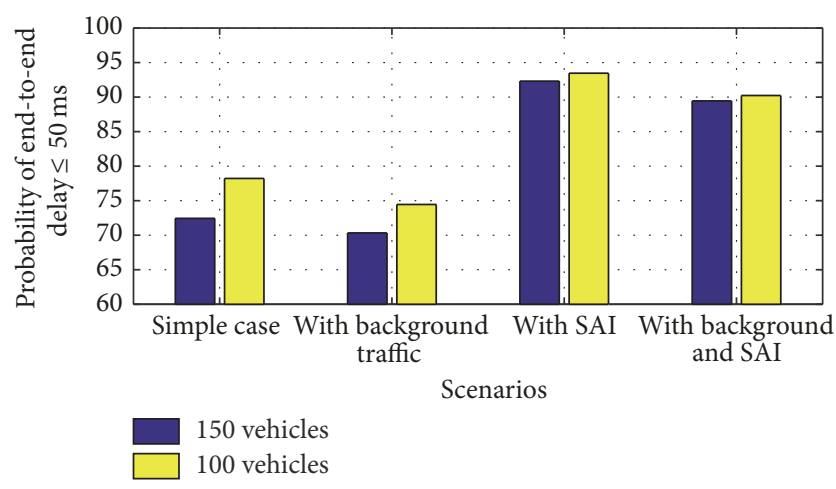

FIGURE 11: Probability of end-to-end delay being $\leq 50 \mathrm{~ms}$ for 100 and 150 vehicles at $40 \mathrm{~km} / \mathrm{h}$ in various scenarios.

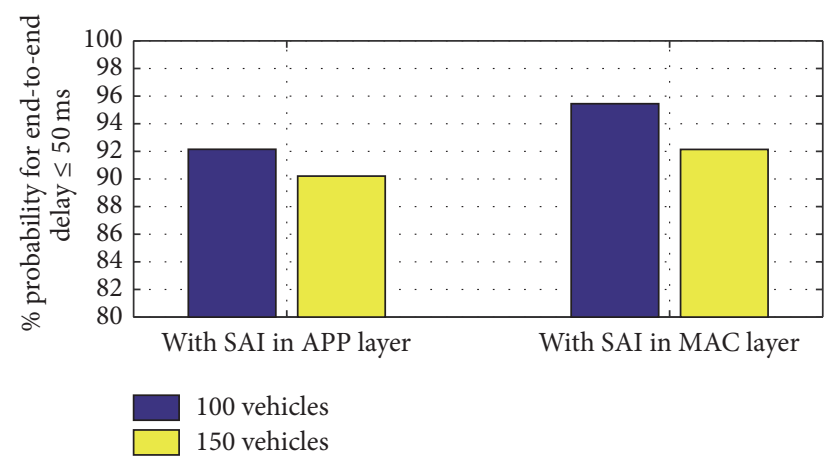

FIGURE 12: Probability of end-to-end delay being $\leq 50 \mathrm{~ms}$ for 100 and 150 vehicles with and without MAC layer inclusion.

\section{Conclusion}

This paper proposes the safety application identifier concept in the form of an algorithm that is tested and analyzed within the impact of vehicular urban multicell radio environment employing multipath fading channels and background traffic. The proposed algorithm uses dynamic adaptation of transmission parameters in order to fulfill stringent vehicular application requirements while minimizing latency and reducing system load.

With the help of extensive system-level simulations, the crucial impact of channel modeling and mobility traces is evident with its influence on the received signal quality, decreasing the probability of end-to-end delay to be $\leq 50 \mathrm{~ms}$ for various awareness ranges by $19 \%$ to $30 \%$ and $9 \%$ to $17 \%$, respectively. Studying the impact of awareness range, it is observed that, with range up to $1000 \mathrm{~m}$ for $1 \mathrm{~Hz}, 500 \mathrm{~m}$ for $2 \mathrm{~Hz}$, and $250 \mathrm{~m}$ for $10 \mathrm{~Hz}$ beacon frequency, the system performed well. With the help of these findings, the SAI algorithm is proposed and implemented. Probability of experiencing an end-to-end delay of less than $100 \mathrm{~ms}$ increased by around $20 \%$, and the downlink goodput decreased significantly from $21.67 \mathrm{Mbps}$ to $13.76 \mathrm{Mbps}$ for 150 vehicles.

Furthermore, the impact of having background traffic is also taken into consideration. Results show that the system is slightly affected by having regular background traffic. However, the probability of end-to-end delay being $\leq 50 \mathrm{~ms}$ still stays satisfactorily above $85 \%$ with the use of SAI. This paper also proposes the inclusion of SAI in MAC layer control elements producing a D2D type of communication within vehicles. After modeling this system, it was found that the same amount of system resources and requirements are met with additional 50 vehicles, increasing the capacity of our LTE vehicular network. Finally in future works, we plan to integrate SAI incorporated within MAC layer in a heterogeneous network with DSRC while exploring the network slicing in $5 \mathrm{G}$ cellular networks for vehicular communications, complementing each other's weaknesses and strengths in order to meet the vehicular network requirements.

\section{Conflicts of Interest}

The authors declare that they have no conflicts of interest.

\section{Acknowledgments}

Marvin Sánchez would like to acknowledge the support of LAMENITEC Erasmus Mundus Program of the European Union to be a guest researcher at GCU. Results were obtained using the EPSRC funded ARCHIE-WeSt High Performance Computer (https://www.archie-west.ac.uk), EPSRC Grant no. EP/K000586/1.

\section{References}

[1] S. Sesia, I. Toufik, and M. Baker, LTE, the UMTS long term evolution: from theory to practice, Wiley Publishing, 2009.

[2] H. Hartenstein and K. P. Laberteaux, "A tutorial survey on vehicular ad hoc networks," IEEE Communications Magazine, vol. 46, no. 6, pp. 164-171, 2008.

[3] D. Jiang and L. Delgrossi, "Towards an international standard for wireless access in vehicular environments," in Proceedings of the 67th IEEE Vehicular Technology Conference (VTC '08), pp. 2036-2040, May 2008.

[4] S. Al-Sultan, M. M. Al-Doori, A. H. Al-Bayatti, and H. Zedan, "A comprehensive survey on vehicular Ad Hoc network," Journal of Network and Computer Applications, vol. 37, no. 1, pp. 380-392, 2014.

[5] K. Tanuja, T. Sushma, M. Bharathi, and K. Arun, "A survey on VANET technologies," International Journal of Computer Applications, vol. 121, no. 18, 2015.

[6] H. Y. Kim, D. M. Kang, J. H. Lee, and T. M. Chung, "A performance evaluation of cellular network suitability for VANET," World Academy of Science, Engineering and Technology, International Science Index, vol. 64, no. 6, pp. 124-127, 2012.

[7] A. Fasbender, M. Gerdes, and S. Smets, "Cellular Networking Technologies in ITS Solutions: Opportunities and Challenges," pp. 1-13, Springer, Berlin, Germany, 2012.

[8] "Cellular coverage and tower map for ee network in glasgow," https://www.cellmapper.net/map.

[9] G. Araniti, C. Campolo, M. Condoluci, A. Iera, and A. Molinaro, "LTE for vehicular networking: a survey," IEEE Communications Magazine, vol. 51, no. 5, pp. 148-157, 2013.

[10] A. Vinel, "3GPP LTE versus IEEE 802.11p/WAVE: which technology is able to support cooperative vehicular safety applications?" IEEE Wireless Communications Letters, vol. 1, no. 2, pp. 125-128, 2012. 
[11] Z. M. Mir and F. Filali, "LTE and IEEE 802.11p for vehicular networking: a performance evaluation," EURASIP Journal on Wireless Communications and Networking, vol. 2014, no. 1, article 89, 2014.

[12] M. Phan, R. Rembarz, and S. Sories, "A capacity analysis for the transmission of event- und cooperative awareness messages in LTE networks," in ITS World Congress 2011, Orlando, USA, Orlando, Florida, USA, 2011.

[13] A. Grzybek, G. Danoy, and P. Bouvry, "Generation of realistic traces for vehicular mobility simulations," in Proceedings of the 2nd ACM International Symposium on Design and Analysis of Intelligent Vehicular Networks and Applications, DIVANet 2012, pp. 131-138, New York, NY, USA, October 2012.

[14] T. Cerqueira and M. Albano, "Routesmobilitymodel: easy realistic mobility simulation using external information services," in Proceedings of the 2015 Workshop on Ns-3, ser. WNS3 '15, pp. 40-46, New York, NY, USA, 2015.

[15] S. Kato, M. Hiltunen, K. Joshi, and R. Schlichting, "Enabling vehicular safety applications over LTE networks," in Proceedings of the 2013 2nd IEEE International Conference on Connected Vehicles and Expo, ICCVE 2013, pp. 747-752, December 2013.

[16] "Public warning system (PWS) requirements (release 13)," 3GPP TS 22.268 V13.0.0, Dec. 2015.

[17] "Overall description; Stage 2 (Release 13)," 3GPP TS 36.300 V13.2.0, Dec. 2015.

[18] G. Remy, S.-M. Senouci, F. Jan, and Y. Gourhant, "LTE4V2X: LTE for a centralized VANET organization," in Proceedings of the 54th Annual IEEE Global Telecommunications Conference: "Energizing Global Communications", GLOBECOM 2011, pp. 16, Houston, TX, USA, December 2011.

[19] Y. Yang, P. Wang, C. Wang, and F. Liu, "An eMBMS based congestion control scheme in cellular-VANET heterogeneous networks," in Proceedings of the 17th IEEE International Conference on Intelligent Transportation Systems (ITSC '14), pp. 1-5, IEEE, Qingdao, China, October 2014.

[20] S. Taha and X. Shen, "A physical-layer location privacypreserving scheme for mobile public hotspots in NEMObased VANETs," IEEE Transactions on Intelligent Transportation Systems, vol. 14, no. 4, pp. 1665-1680, 2013.

[21] G. T. V12.9.0, Evolved Universal Terrestrial Radio Access (EUTRA), Medium Access Control (MAC) protocol specification (Release 12), 3GPP, 2016.

[22] A. Bazzi, B. M. Masini, and A. Zanella, "Performance analysis of V2v beaconing using LTE in direct mode with full duplex radios," IEEE Wireless Communications Letters, vol. 4, no. 6, pp. 685-688, 2015.

[23] D. Tsolkas, E. Liotou, N. Passas, and L. Merakos, LTE-a Access, Core, and Protocol Architecture for D2D Communication, S. Mumtaz and J. Rodriguez, Eds., Springer International Publishing, 2014.

[24] Y. Bi, H. Shan, X. S. Shen, N. Wang, and H. Zhao, "A multihop broadcast protocol for emergency message dissemination in urban vehicular ad hoc networks," IEEE Transactions on Intelligent Transportation Systems, vol. 17, no. 3, pp. 736-750, 2016.

[25] "Vehicle safety communications project task 3 final report," Tech. Rep., US Department of Transportation, 2005.

[26] Intelligent transport systems (ITS); basic set of applications; Part2: specification of cooperative awareness basic service, ETSI TS 102 637-2 V1.2.1, 2011.
[27] Intelligent transport systems (ITS); basic set of applications; part3: specifications of decentralized environmental notification basic service, ETSI TS 102 637-3 V1.1.1, 2010.

[28] C. L. Robinson, D. Caveney, L. Caminiti, G. Baliga, K. Laberteaux, and P. R. Kumar, "Efficient message composition and coding for cooperative vehicular safety applications," IEEE Transactions on Vehicular Technology, vol. 56, no. 6 I, pp. 32443255, 2007.

[29] D. Caveney, "Cooperative vehicular safety applications: collision avoidance enabled through geospatial positioning and intervehicular communications," IEEE Control Systems Magazine, vol. 30, no. 4, pp. 38-53, 2010.

[30] W. Sun, T. Fu, Y. Su, F. Xia, and J. Ma, "ODAM-C: an improved algorithm for vehicle Ad Hoc network," in Proceedings of the 2011 IEEE International Conference on Internet of Things, iThings 2011 and 4th IEEE International Conference on Cyber, Physical and Social Computing, CPSCom 2011, pp. 152-156, October 2011.

[31] S. Ansari, T. Boutaleb, C. Gamio, S. Sinanovic, I. Krikidis, and M. Sanchez, "Vehicular Safety Application Identifier algorithm for LTE VANET server," in Proceedings of the 8th International Congress on Ultra Modern Telecommunications and Control Systems and Workshops, ICUMT 2016, pp. 37-42, October 2016.

[32] Intelligent transport systems (ITS): communications architecture, ETSI EN 302665 V1.1.1, 2010.

[33] S. Sinanović, H. Burchardt, H. Haas, and G. Auer, "Sum rate increase via variable interference protection," IEEE Transactions on Mobile Computing, vol. 11, no. 12, pp. 2121-2132, 2012.

[34] E-UTRA Base Station (BS) radio transmission and reception (Release 12), 3GPP TS 36.104 V12.10.0, 2016.

[35] "Evolved Universal Terrestrial Radio Access Network (EUTRAN), Stage 2 functional specification of User Equipment (UE) positioning in E-UTRAN (Release 9)".

[36] G. T. V13.1.0, Evolved Universal Terrestrial Radio Access (EUTRA), Medium Access Control (MAC) protocol specification (Release 13), 3GPP, 2016.

[37] "Model library release ns-3.2," Ns-3 network simulator, 2015, https://www.nsnam.org/docs/models/html/lte-design.html.

[38] G. Piro, N. Baldo, and M. Miozzo, "An LTE module for the ns-3 network simulator," in Proceedings of the 4th International ICST Conference on Simulation Tools and Techniques, 422, ser. SIMUTools '11. ICST, 415 pages, March 2011.

[39] L. Chunjian, Efficient antenna patterns for three-sector WCDMA system, Master of Science Thesis, Chalmers University of Technology, Göteborg, Sweden, 2003.

[40] Evolved universal terrestrial radio access (E-UTRA); physical layer procedures, 3GPP TS 36.213 V13.0.1, 2016.

[41] D. Kimura and H. Seki, "Inter-cell interference coordination (ICIC) technology," Fujitsu Scientific \& Technical Journal, vol. 48, no. 1, pp. 89-94, 2012.

[42] Physical layer; measurements (release 13), 3GPP TS 36.214 V13.0.0, 2015.

[43] Evolved universal terrestrial radio access (E-UTRA); radio resource control (RRC); protocol specification, 3GPP TS 36.213 V13.0.0, 2015.

[44] Y. S. Cho, W. Y. Yang, and C. Kang, IMO-OFDM Wireless Communications with MATLAB, John Wiley \& Sons, 2010.

[45] J. Archer and K. Vogel, The traffic safety problem in urban areas, 2000.

[46] “Urban crashes," Insurance Institute for Highway Safety, 1999. 
[47] J. Klaue, B. Rathke, and A. Wolisz, EvalVid - A Framework for Video Transmission and Quality Evaluation, P. Kemper and W. H. Sanders, Eds., Springer, Berlin, Germany, 2003.

[48] S. Ucar, S. C. Ergen, and O. Ozkasap, "Multi-hop cluster based IEEE 802.11p and LTE hybrid architecture for VANET safety message dissemination," IEEE Transactions on Vehicular Technology, vol. PP, no. 99, pp. 1-1, 2015.

[49] L. Ward and M. Simon, "Intelligent transportation systems using IEEE 802.11p," Rohde and Schwarz - Application Note, 2015. 


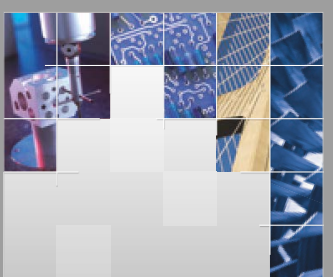

\section{Enfincering}
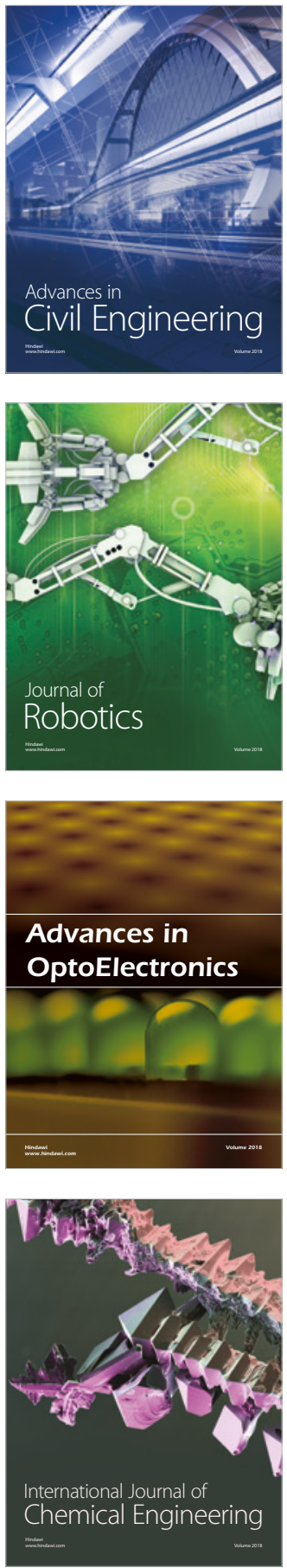

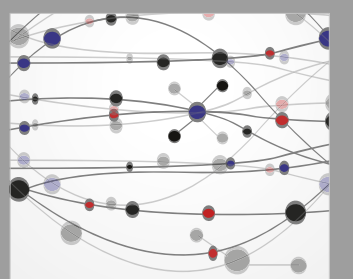

\section{Rotating \\ Machinery}

The Scientific World Journal

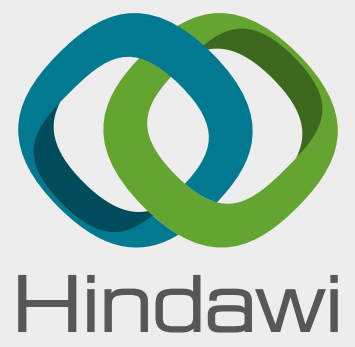

Submit your manuscripts at

www.hindawi.com
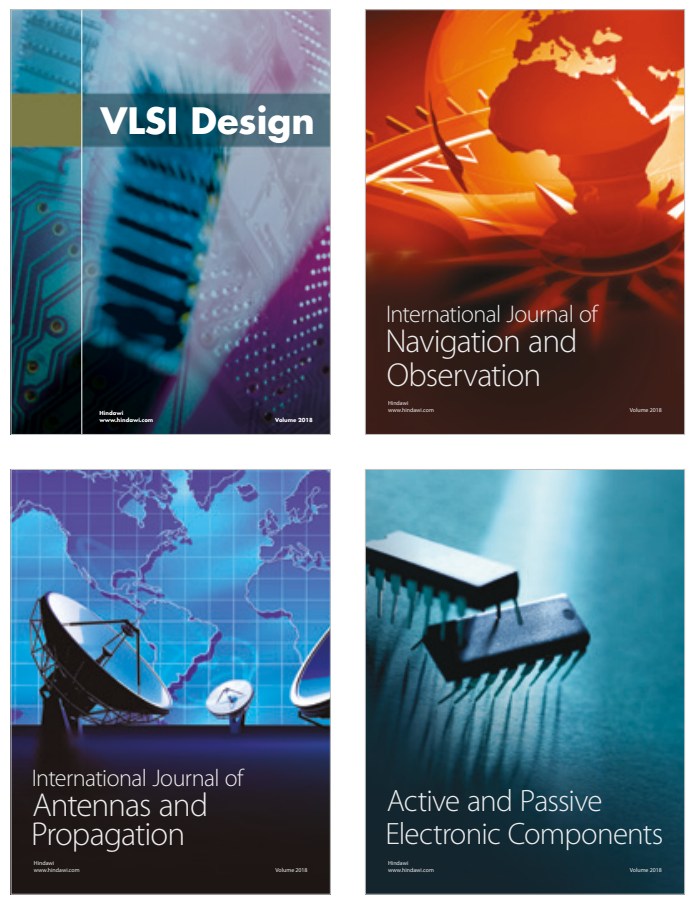
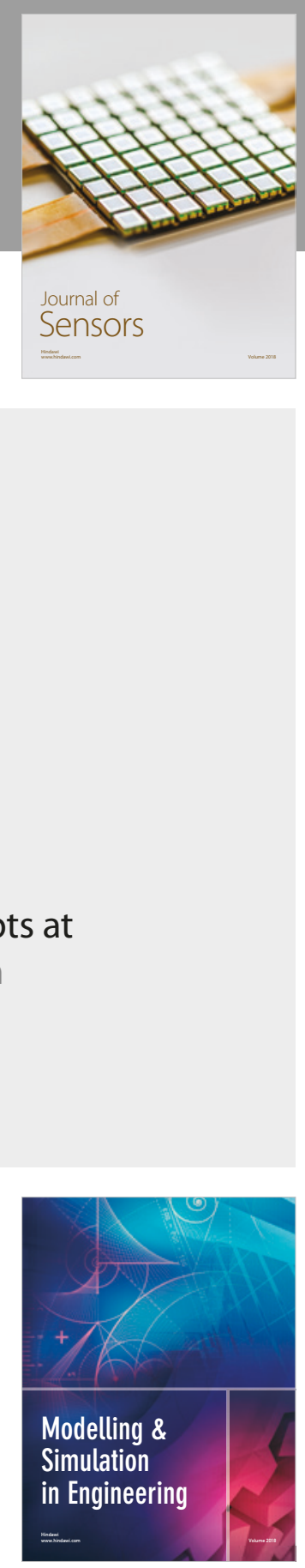

\section{Advances \\ Multimedia}
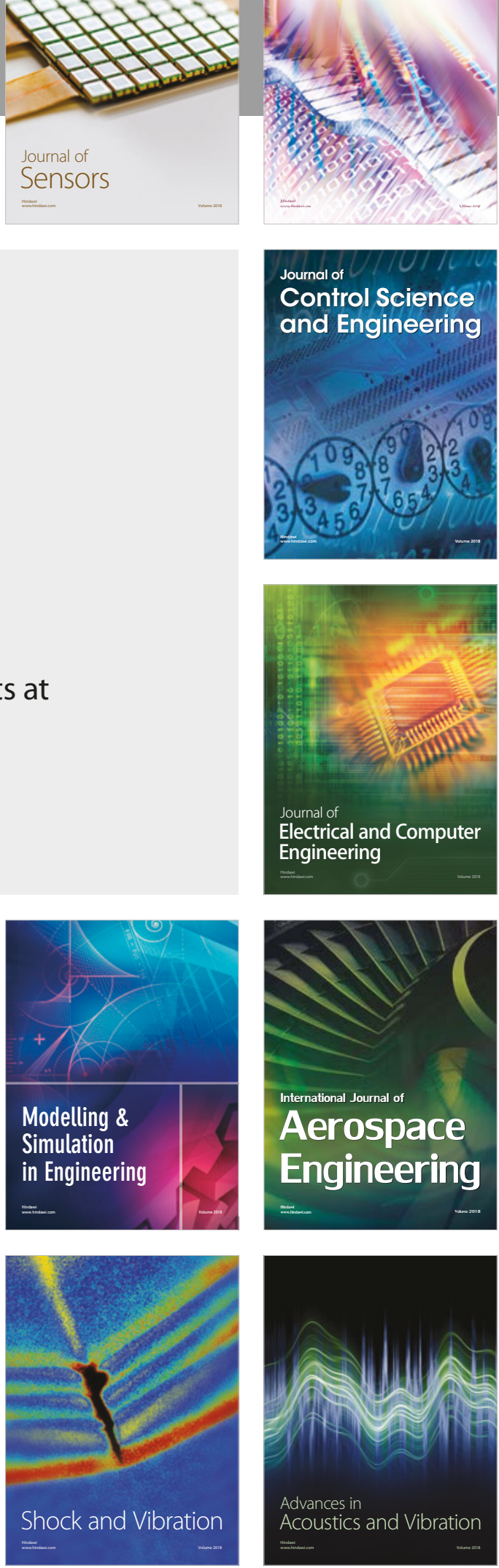